\title{
A Practical Asymmetric Synthesis of trans-4,5-Benzhydrindan-1-ones as a Precursor of A-Nor B-Aromatic Steroidal Compounds
}

\author{
Yuji Matsuya, Seiji Masuda, Takakatsu Itoh, Taiki Murai, and Hideo Nemoto* \\ Faculty of Pharmaceutical Sciences, Toyama Medical and Pharmaceutical University, \\ 2630 Sugitani, Toyama 930-0194, Japan \\ nemotoh@ms.toyama-mpu.ac.jp
}

\section{Supporting Information}

\section{Contents}

Synthetic procedure and characterization data for the compounds 6-9 (S2-S4)

Identification of $\mathbf{2 a}$ and ${ }^{1} \mathrm{H}$ NMR data for the compounds 11a-14a (S5)

Synthetic procedure and characterization data for the compounds 15-18 (S6-S7)

References (S7)

${ }^{1} \mathrm{H}$ and ${ }^{13} \mathrm{C}$ NMR spectra for the compounds $2 \mathbf{a}, \mathbf{1 1 a}-\mathbf{1 4 a}, \mathbf{1 b}-\mathbf{5 b}, \mathbf{5 c}$, and 11b-14b (S8S33) 
General Remarks. All nonaqueous reactions were carried out under Ar atmosphere. Reagents were purchased from commercial sources and used as received. Anhydrous solvents were obtained from commercial sources or prepared by distillation over $\mathrm{CaH}_{2}$ or $\mathrm{P}_{2} \mathrm{O}_{5}$. Chemical shifts of ${ }^{1} \mathrm{H}$ and ${ }^{13} \mathrm{C}$ NMR spectra are given in ppm based on solvent signals. Ionization method of mass spectrometry is electron impact (EI, $70 \mathrm{eV}$ ). Melting points are uncorrected. Column chromatography was carried out by employing Cica Silica Gel $60 \mathrm{~N}$ (spherical, neutral, 40-50 $\mu \mathrm{m}$ or 63-210 $\mu \mathrm{m}$ ). Experimental details of the compounds $\mathbf{2 a - 5 a}, \mathbf{1 0}$, and 19 have already been given in our previous communication. ${ }^{1}$

\section{4-Methoxy-1-[3-(tetrahydropyran-2-yloxy)prop-1-yl]benzocyclobutene-1-}

carbonitrile (6). A solution of LDA $(3.38 \mathrm{mmol})$ in THF $(10 \mathrm{ml})$ was cooled to $-78^{\circ} \mathrm{C}$, and the benzocyclobutene $\mathbf{1 a}(500 \mathrm{mg}, 3.14 \mathrm{mmol})$ in THF (10 ml) was added dropwise. After stirring for $10 \mathrm{~min}$, the alkyl bromide $(0.77 \mathrm{~g}, 3.45 \mathrm{mmol})$ in THF $(5 \mathrm{ml})$ was added, and the mixture was stirred at $0^{\circ} \mathrm{C}$ for $1 \mathrm{~h}$. The reaction was quenched by sat. $\mathrm{NaHCO}_{3}$, and extracted with $\mathrm{Et}_{2} \mathrm{O}$, and then dried over $\mathrm{MgSO}_{4}$. Removal of the solvent left a residue, which was chromatographed on silica gel to afford $6(760 \mathrm{mg}, 81 \%)$ as a colorless oil. ${ }^{1} \mathrm{H}$ NMR $\left(270 \mathrm{MHz}, \mathrm{CDCl}_{3}\right): \delta 7.10(\mathrm{~d}, J=7.7 \mathrm{~Hz}, 1 \mathrm{H}), 6.80(\mathrm{~d}, J=7.7$ $\mathrm{Hz}, 1 \mathrm{H}), 6.70$ (s, 1H), 4.56 (brs, 1H), 3.76 (brs, 5H), 3.60 (d, $J=14 \mathrm{~Hz}, 1 \mathrm{H}), 3.48$ $3.43(\mathrm{~m}, 2 \mathrm{H}), 3.20(\mathrm{~d}, J=14 \mathrm{~Hz}, 1 \mathrm{H}), 1.99-1.51(\mathrm{~m}, 10 \mathrm{H}) ;{ }^{13} \mathrm{C}$ NMR $(75 \mathrm{MHz}$, $\left.\mathrm{CDCl}_{3}\right): \delta 161.0,142.1,135.3,122.9,122.0,114.9,109.3,98.9,77.2,66.7,62.5,55.6$, 42.2, 34.6, 30.8, 26.8, 25.6, 19.8; IR (neat) $\mathrm{cm}^{-1}: 2231$; MS (EI): $\mathrm{m} / z 301\left(\mathrm{M}^{+}\right)$; HRMS (EI) calcd for $\mathrm{C}_{18} \mathrm{H}_{23} \mathrm{NO}_{3}: 301.1678\left(\mathrm{M}^{+}\right)$, found: 301.1683 .

\section{1-(1-Hydroxy-2-penten-5-yl)-4-methoxybenzocyclobutene-1-carbonitrile (7). The} THP ether $6(1.86 \mathrm{~g}, 6.17 \mathrm{mmol})$ was dissolved in $\mathrm{MeOH}(30 \mathrm{ml})$, and $10 \% \mathrm{HCl}$ solution $(3.6 \mathrm{ml})$ was added to the solution. After stirring at room temperature for $2 \mathrm{~h}$, the solvent was evaporated in vacuo, and the residue was suspended in sat. $\mathrm{NaHCO}_{3}$. The organic layer obtained by $\mathrm{Et}_{2} \mathrm{O}$ extraction was washed with brine, dried, and evaporated to give the alcohol. This crude alcohol was directly subjected to the standard Swern oxidation condition to afford the corresponding aldehyde as a pale yellow oil, which was used for the next reaction without column chromatography. To a suspension of sodium hydride $(60 \%, 300 \mathrm{mg}, 7.4 \mathrm{mmol})$ in THF $(20 \mathrm{ml})$ was added the HornerEmmons reagent $(1.5 \mathrm{ml}, 7.4 \mathrm{mmol})$, and the mixture was stirred at $0^{\circ} \mathrm{C}$ for $0.5 \mathrm{~h}$. A solution of the crude aldehyde in THF $(12 \mathrm{ml})$ was then added, and stirring continued 
overnight. The reaction mixture was diluted with $\mathrm{H}_{2} \mathrm{O}$ and extracted with $\mathrm{Et}_{2} \mathrm{O}$. The organic layer was washed with brine, dried over $\mathrm{MgSO}_{4}$, and evaporated to leave a residue, which was purified by silica gel column chromatography to afford the ethyl ester (730 mg, $42 \%$ in 3 steps) as a pale yellow oil. ${ }^{1} \mathrm{H}$ NMR $\left(270 \mathrm{MHz}, \mathrm{CDCl}_{3}\right): \delta 7.10$ $(\mathrm{d}, J=8.0 \mathrm{~Hz}, 1 \mathrm{H}), 6.95(\mathrm{~m}, 1 \mathrm{H}), 6.83(\mathrm{~d}, J=8.0 \mathrm{~Hz}, 1 \mathrm{H}), 6.70(\mathrm{~s}, 1 \mathrm{H}), 5.90(\mathrm{~m}, 1 \mathrm{H})$, 4.17 (q, $J=7.4 \mathrm{~Hz}, 2 \mathrm{H}), 3.81-3.67(\mathrm{~m}, 4 \mathrm{H}), 3.28-3.21(\mathrm{~m}, 1 \mathrm{H}), 2.58-2.50(\mathrm{~m}, 2 \mathrm{H})$, $2.50-2.08(\mathrm{~m}, 2 \mathrm{H}), 1.28(\mathrm{t}, J=7.4 \mathrm{~Hz}, 3 \mathrm{H})$; IR (neat) $\mathrm{cm}^{-1}: 2231,1716$; MS (EI): $\mathrm{m} / z$ $285\left(\mathrm{M}^{+}\right)$; HRMS (EI) calcd for $\mathrm{C}_{17} \mathrm{H}_{19} \mathrm{NO}_{3}: 285.1365\left(\mathrm{M}^{+}\right)$, found: 285.1331.

DIBAL (0.95 M in hexane, $5.94 \mathrm{ml}, 5.64 \mathrm{mmol})$ was added to a solution of the ethyl ester $(730 \mathrm{mg}, 2.57 \mathrm{mmol})$ in $\mathrm{Et}_{2} \mathrm{O}(25 \mathrm{ml})$ at $-78^{\circ} \mathrm{C}$, and the reaction continued at the same temperature for $2 \mathrm{~h}$. The reaction was quenched with $10 \% \mathrm{H}_{2} \mathrm{SO}_{4}$, and the mixture was vigorously stirred at room temperature for $0.5 \mathrm{~h}$. The precipitates were removed by filtration, and the filtrate was extracted with $\mathrm{CH}_{2} \mathrm{Cl}_{2}$, dried, and then concentrated. The residue was chromatographed on silica gel to give the alcohol 7 (393 $\mathrm{mg}, 63 \%)$ as a colorless oil. ${ }^{1} \mathrm{H}$ NMR $\left(270 \mathrm{MHz}, \mathrm{CDCl}_{3}\right): \delta 7.12(\mathrm{~d}, J=8.0 \mathrm{~Hz}, 1 \mathrm{H}), 6.82(\mathrm{~d}, J=8.0$ $\mathrm{Hz}, 1 \mathrm{H}), 6.72(\mathrm{~s}, 1 \mathrm{H}), 5.75-5.61(\mathrm{~m}, 2 \mathrm{H}), 4.10-4.05(\mathrm{~m}, 2 \mathrm{H}), 3.88-3.65(\mathrm{~m}, 4 \mathrm{H})$, $3.23(\mathrm{~d}, J=14 \mathrm{~Hz}, 1 \mathrm{H}), 2.44-2.40(\mathrm{~m}, 2 \mathrm{H}), 2.17-1.93(\mathrm{~m}, 2 \mathrm{H})$; IR (neat) $\mathrm{cm}^{-1}: 3413$, 2230; MS (EI): $m / z 243\left(\mathrm{M}^{+}\right)$; HRMS (EI) calcd for $\mathrm{C}_{15} \mathrm{H}_{17} \mathrm{NO}_{2}: 243.1259\left(\mathrm{M}^{+}\right)$, found: 243.1295 .

4-Methoxy-1-(1,2,3-trihydroxypent-5-yl)benzocyclobutene-1-carbonitrile (8). The allyl alcohol 7 (79 $\mathrm{mg}, 0.32 \mathrm{mmol})$ was dihydroxylated under the standard Sharpless $\mathrm{AD}$ condition using (DHQD) $)_{2} \mathrm{AQN}$ as a chiral ligand in tert-BuOH $(5 \mathrm{ml})$ and $\mathrm{H}_{2} \mathrm{O}(5$ $\mathrm{ml}$ ) at room temperature for $12 \mathrm{~h}$. Purification by silica gel column chromatography afforded the triol $8(78 \mathrm{mg}, 87 \%)$ as a colorless oil. ${ }^{1} \mathrm{H}$ NMR $\left(270 \mathrm{MHz}, \mathrm{CDCl}_{3}\right): \delta 7.12$ $(\mathrm{d}, J=8.0 \mathrm{~Hz}, 1 \mathrm{H}), 6.81(\mathrm{~d}, J=8.0 \mathrm{~Hz}, 1 \mathrm{H}), 6.72(\mathrm{~s}, 1 \mathrm{H}), 3.89-3.58(\mathrm{~m}, 8 \mathrm{H}), 3.28-$ $3.23(\mathrm{~m}, 1 \mathrm{H}), 2.67$ (d, $J=5.3 \mathrm{~Hz}, 1 \mathrm{H}), 2.42$ (d, $J=3.3 \mathrm{~Hz}, 1 \mathrm{H}), 2.28-1.85$ (m, 5H); IR (neat) $\mathrm{cm}^{-1}: 3415,2233$; MS (EI): $\mathrm{m} / z 277\left(\mathrm{M}^{+}\right)$; HRMS (EI) calcd for $\mathrm{C}_{15} \mathrm{H}_{19} \mathrm{NO}_{4}$ : $277.1314\left(\mathrm{M}^{+}\right)$, found: 277.1307.

\section{1-[3-(2,2-Dimethyl-1,3-dioxolan-4-yl)-3-hydroxyprop-1-yl]-4-}

methoxybenzocyclobutene-1-carbonitrile (9). To a solution of the triol 8 (78 $\mathrm{mg}, 0.28$ $\mathrm{mmol})$ and 2-methoxypropene $(0.081 \mathrm{ml}, 0.84 \mathrm{mmol})$ in anhydrous DMF $(5 \mathrm{ml})$ was added $5 \mathrm{M} \mathrm{HCl} / \mathrm{MeOH}$ ( 1 drop) at $0^{\circ} \mathrm{C}$. After continuous stirring for $12 \mathrm{~h}$, the mixture was diluted with sat. $\mathrm{NaHCO}_{3}$, and extracted with $\mathrm{CHCl}_{3}$. The organic layer was dried 
and evaporated to leave a residue, which was chromatographed on silica gel to afford the acetal 9 (20 mg, 22\%) as a colorless oil. ${ }^{1} \mathrm{H}$ NMR $\left(270 \mathrm{MHz}, \mathrm{CDCl}_{3}\right): \delta 7.12(\mathrm{~d}, J=$ $8.0 \mathrm{~Hz}, 1 \mathrm{H}), 6.83(\mathrm{~d}, J=8.0 \mathrm{~Hz}, 1 \mathrm{H}), 6.73(\mathrm{~s}, 1 \mathrm{H}), 4.09-4.01(\mathrm{~m}, 2 \mathrm{H}), 3.80-3.53$ (m, $5 \mathrm{H}), 3.29-3.23(\mathrm{~m}, 1 \mathrm{H}), 2.40-1.38(\mathrm{~m}, 11 \mathrm{H})$; IR (neat) $\mathrm{cm}^{-1}: 3424,2232$; MS (EI): $\mathrm{m} / z 317\left(\mathrm{M}^{+}\right)$; HRMS (EI) calcd for $\mathrm{C}_{18} \mathrm{H}_{23} \mathrm{NO}_{4}: 317.1627\left(\mathrm{M}^{+}\right)$, found: 317.1652 . 
The compounds 11a-14a were synthesized in the yields indicated in Scheme 4, according to the same procedure as described for the synthesis of $\mathbf{1 1 b}-\mathbf{1 4 b}$ in the Experimental Section. The compound 2a was also synthesized from 14a in the same way, which was confirmed to be wholly identical with that synthesized and characterized previously. ${ }^{1}$ A comparison between the NMR spectra of $\mathbf{2 a}$ obtained from 14a and $2 \mathbf{a}$ synthesized through Scheme $3^{1}$ is provided at the pages S22-S23. The followings are ${ }^{1} \mathrm{H}$ NMR data of 11a-14a, whose similarity to those of $\mathbf{1 1 b}-\mathbf{1 4 b}$ well supports the structures.

4-Methoxybenzocyclobutene-1-carbaldehyde (11a). ${ }^{1} \mathrm{H} \mathrm{NMR}\left(500 \mathrm{MHz}, \mathrm{CDCl}_{3}\right)$ : $\delta$ $9.68(\mathrm{~d}, J=3.8 \mathrm{~Hz}, 1 \mathrm{H}), 7.08(\mathrm{~d}, J=8.1 \mathrm{~Hz}, 1 \mathrm{H}), 6.82(\mathrm{dd}, J=8.1,1.3 \mathrm{~Hz}, 1 \mathrm{H}), 6.66(\mathrm{~d}$, $J=1.3 \mathrm{~Hz}, 1 \mathrm{H}), 4.16-4.14(\mathrm{~m}, 1 \mathrm{H}), 3.78(\mathrm{~s}, 3 \mathrm{H}), 3.40-3.32(\mathrm{~m}, 2 \mathrm{H})$.

1-(2,2-Dibromoethenyl)-4-methoxybenzocyclobutene (12a). ${ }^{1} \mathrm{H}$ NMR (500 MHz, $\left.\mathrm{CDCl}_{3}\right): \delta 7.02(\mathrm{~d}, J=8.1 \mathrm{~Hz}, 1 \mathrm{H}), 6.77(\mathrm{dd}, J=8.1,1.7 \mathrm{~Hz}, 1 \mathrm{H}), 6.68(\mathrm{~d}, J=1.7 \mathrm{~Hz}$, $1 \mathrm{H}), 6.62(\mathrm{~d}, J=9.0 \mathrm{~Hz}, 1 \mathrm{H}), 4.24-4.21(\mathrm{~m}, 1 \mathrm{H}), 3.78(\mathrm{~s}, 3 \mathrm{H}), 3.53(\mathrm{dd}, J=14,5.5 \mathrm{~Hz}$, 1H), $2.96(\mathrm{dd}, J=14,2.1 \mathrm{~Hz}, 1 \mathrm{H})$.

1-Ethynyl-4-methoxybenzocyclobutene (13a). ${ }^{1} \mathrm{H} \mathrm{NMR}\left(500 \mathrm{MHz}, \mathrm{CDCl}_{3}\right): \delta 7.10(\mathrm{~d}$, $J=8.1 \mathrm{~Hz}, 1 \mathrm{H}), 6.82(\mathrm{dd}, J=8.1,2.1 \mathrm{~Hz}, 1 \mathrm{H}), 6.71(\mathrm{~d}, J=2.1 \mathrm{~Hz}, 1 \mathrm{H}), 4.15-4.13(\mathrm{~m}$, $1 \mathrm{H}), 3.79$ (s, 3H), 3.56 (dd, $J=14,5.2 \mathrm{~Hz}, 1 \mathrm{H}), 3.23(\mathrm{dd}, J=14,2.6 \mathrm{~Hz}, 1 \mathrm{H}), 2.29$ (d, $J$ $=2.6 \mathrm{~Hz}, 1 \mathrm{H})$.

\section{1-[3-(2,2-Dimethyl-1,3-dioxolan-4-yl)-3-hydroxy-1-propyn-1-yl]-4-}

methoxybenzocyclobutene (14a). ${ }^{1} \mathrm{H}$ NMR $\left(300 \mathrm{MHz}, \mathrm{CDCl}_{3}\right): \delta 7.03(\mathrm{~d}, J=8.1 \mathrm{~Hz}$, $1 \mathrm{H}), 6.78(\mathrm{dd}, J=8.1,1.7 \mathrm{~Hz}, 1 \mathrm{H}), 6.67(\mathrm{~d}, J=1.7 \mathrm{~Hz}, 1 \mathrm{H}), 4.52-3.83(\mathrm{~m}, 5 \mathrm{H}), 3.77$ $(\mathrm{s}, 3 \mathrm{H}), 3.52(\mathrm{dd}, J=14,5.5 \mathrm{~Hz}, 1 \mathrm{H}), 3.19-3.14(\mathrm{~m}, 1 \mathrm{H}), 2.53$ and $2.38(\mathrm{br}, 1 \mathrm{H}), 1.44$ and $1.43(\mathrm{~s}, 3 \mathrm{H}), 1.36(\mathrm{~s}, 3 \mathrm{H})$. 
Preparation of the Compound 15. To a solution of 1-hexyne $(0.22 \mathrm{ml}, 1.9 \mathrm{mmol})$ in THF (4 ml) was added $n$-BuLi (1.6 M in hexane, $1.2 \mathrm{ml}, 1.92 \mathrm{mmol})$ at $-78^{\circ} \mathrm{C}$. After stirring at $-78^{\circ} \mathrm{C}$ for $20 \mathrm{~min}$, a solution of $(R)$-2,2-dimethyl-1,3-dioxolane-4carbaldehyde $(500 \mathrm{mg}, 3.8 \mathrm{mmol})$ in THF ( $3 \mathrm{ml})$ was added to the mixture, and the resulting solution was stirred at $-78^{\circ} \mathrm{C}$ for $40 \mathrm{~min}$. The reaction was quenched by addition of sat. $\mathrm{NH}_{4} \mathrm{Cl}$, and the aqueous mixture was extracted with $\mathrm{CH}_{2} \mathrm{Cl}_{2}$. After drying over $\mathrm{MgSO}_{4}$, the solvent was evaporated to leave a residue, which was chromatographed on silica gel to afford an adduct (162 $\mathrm{mg}, 41 \%)$ as a mixture of diastereomers. This alkynyl alcohol was hydrogenated as follows; a mixture of the alkyne $(400 \mathrm{mg}, 1.9 \mathrm{mmol})$ and $10 \% \mathrm{Pd} / \mathrm{C}(88 \mathrm{mg})$ in $\mathrm{MeOH}(10 \mathrm{ml})$ was vigorously stirred under $\mathrm{H}_{2}$ atmosphere for $9 \mathrm{~h}$. The catalyst was removed by filtration, and the filtrate was concentrated to give the saturated alcohol $(355 \mathrm{mg}, 88 \%)$ as a crude form. The alcohol thus obtained (60 mg, $0.3 \mathrm{mmol}$ ) was dissolved in $\mathrm{CH}_{2} \mathrm{Cl}_{2}(5 \mathrm{ml})$, and $\mathrm{NMO}$ (66 mg, $0.6 \mathrm{mmol}$ ), molecular sieves 4A (112 mg), and TPAP (30 mg, $0.08 \mathrm{mmol}$ ) were added successively. The mixture was stirred at room temperature for $12 \mathrm{~h}$, and insoluble materials were filtered off. The filtrate was concentrated and subjected to column chromatography to afford the ketone 15 (37 mg, 62\%) as a colorless oil. ${ }^{1} \mathrm{H}$ NMR (270 $\left.\mathrm{MHz}, \mathrm{CDCl}_{3}\right): \delta 4.41(\mathrm{dd}, J=7.9,5.6 \mathrm{~Hz}, 1 \mathrm{H}), 4.17(\mathrm{dd}, J=8.6,7.9 \mathrm{~Hz}, 1 \mathrm{H}), 3.95$ (dd, $J=8.6,5.6 \mathrm{~Hz}, 1 \mathrm{H}), 2.58(\mathrm{t}, J=7.3 \mathrm{~Hz}, 2 \mathrm{H}), 1.54-1.26(\mathrm{~m}, 8 \mathrm{H}), 1.46(\mathrm{~s}, 3 \mathrm{H}), 1.37(\mathrm{~s}$, $3 \mathrm{H}), 0.86(\mathrm{t}, J=6.6 \mathrm{~Hz}, 3 \mathrm{H}) ;{ }^{13} \mathrm{C} \mathrm{NMR}\left(75 \mathrm{MHz}, \mathrm{CDCl}_{3}\right): \delta 210.9,110.9,80.4,76.7$, 38.8, 31.8, 29.0, 26.3, 25.2, 23.1, 22.7, 14.3; IR (neat) $\mathrm{cm}^{-1}: 1710$; MS (EI): $\mathrm{m} / z 214$ $\left(\mathrm{M}^{+}\right)$; HRMS (EI) calcd for $\mathrm{C}_{12} \mathrm{H}_{22} \mathrm{O}_{3}: 214.1569\left(\mathrm{M}^{+}\right)$, found: 214.1565 .

Preparation of the Compound 16. To a solution of $(R)$-2,2-dimethyl-1,3-dioxolane-4carbaldehyde (500 mg, $3.8 \mathrm{mmol})$ in THF (12 ml) was added 3-phenylmagnesium bromide (0.6 M in THF, $19 \mathrm{ml}, 11 \mathrm{mmol})$ at $-78^{\circ} \mathrm{C}$, and the mixture was stirred at the same temperature for $2 \mathrm{~h}$. After addition of sat. $\mathrm{NH}_{4} \mathrm{Cl}$, the aqueous mixture was extracted with $\mathrm{Et}_{2} \mathrm{O}$ and dried over $\mathrm{MgSO}_{4}$. Evaporation of the solvent gave a residue, which was chromatographed on silica gel to afford an adduct (592 $\mathrm{mg}, 63 \%)$ as a mixture of diastereomers. This alcohol $(550 \mathrm{mg}, 2.2 \mathrm{mmol})$ was subjected to the TPAP oxidation, according to the same procedure as mentioned above, to afford the ketone $\mathbf{1 6}$ (400 mg, 73\%) as a colorless oil. ${ }^{1} \mathrm{H}$ NMR (270 MHz, $\left.\mathrm{CDCl}_{3}\right): \delta 7.29-7.14(\mathrm{~m}, 5 \mathrm{H})$, $4.41(\mathrm{dd}, J=7.9,5.6 \mathrm{~Hz}, 1 \mathrm{H}), 4.17(\mathrm{dd}, J=8.6,7.9 \mathrm{~Hz}, 1 \mathrm{H}), 3.95(\mathrm{dd}, J=8.6,5.6 \mathrm{~Hz}$, $1 \mathrm{H}), 2.64-2.58(\mathrm{~m}, 4 \mathrm{H}), 1.96-1.85(\mathrm{~m}, 2 \mathrm{H}), 1.53(\mathrm{~s}, 3 \mathrm{H}), 1.36(\mathrm{~s}, 3 \mathrm{H}) ;{ }^{13} \mathrm{C} \mathrm{NMR}(75$ 
$\left.\mathrm{MHz}, \mathrm{CDCl}_{3}\right): \delta 210.6,141.5,128.5,128.4,126.0,111.0,80.4,66.7,38.0,35.2,26.2$, 25.2, 24.6; IR (neat) $\mathrm{cm}^{-1}: 1710$; MS (EI): $\mathrm{m} / z 248\left(\mathrm{M}^{+}\right)$; HRMS (EI) calcd for $\mathrm{C}_{15} \mathrm{H}_{20} \mathrm{O}_{3}$ : $248.1412\left(\mathrm{M}^{+}\right)$, found: 248.1410 .

The Reaction of the Compounds 15 and 16 with Grignard Reagent; Formation of the Compounds 17 and 18. The same procedure as described in the transformation of the compounds $\mathbf{2}$ to $\mathbf{3}$ (Experimental Section) was applied for the compounds $\mathbf{1 5}$ and $\mathbf{1 6 .}$ The ketone $\mathbf{1 5}$ gave anti-17 in 80\% yield, and the ketone $\mathbf{1 6}$ gave a mixture of anti-18 and syn-18 (ratio 92/8) in 86\% yield as a colorless oil, respectively. 17: ${ }^{1} \mathrm{H}$ NMR (270 $\left.\mathrm{MHz}, \mathrm{CDCl}_{3}\right): \delta 5.07(\mathrm{~s}, 1 \mathrm{H}), 4.99(\mathrm{~s}, 1 \mathrm{H}), 4.23(\mathrm{t}, J=6.2 \mathrm{~Hz}, 1 \mathrm{H}), 3.99-3.89(\mathrm{~m}, 2 \mathrm{H})$, $1.76(\mathrm{~s}, 3 \mathrm{H}), 1.53(\mathrm{bs}, 1 \mathrm{H}), 1.42-1.24(\mathrm{~m}, 10 \mathrm{H}), 1.39$ (s, 3H), $1.34(\mathrm{~s}, 3 \mathrm{H}), 0.85$ (t, $J=$ $6.6 \mathrm{~Hz}, 3 \mathrm{H}) ;{ }^{13} \mathrm{C} \mathrm{NMR}\left(75 \mathrm{MHz}, \mathrm{CDCl}_{3}\right): \delta 109.5,107.0,104.5,88.6,82.9,67.0,32.9$, 32.5, 30.7, 28.37, 28.34, 23.1, 22.6, 15.2, 14.0; IR (neat) $\mathrm{cm}^{-1}: 3350 ; \mathrm{MS}$ (EI): $\mathrm{m} / z .256$ $\left(\mathrm{M}^{+}\right)$; HRMS (EI) calcd for $\mathrm{C}_{15} \mathrm{H}_{28} \mathrm{O}_{3}: 256.2038\left(\mathrm{M}^{+}\right)$, found: 256.2034 . 18 (as a mixture of diastereomers): ${ }^{1} \mathrm{H}$ NMR $\left(270 \mathrm{MHz}, \mathrm{CDCl}_{3}\right): \delta 7.28-7.12(\mathrm{~m}, 5 \mathrm{H}), 5.07(\mathrm{~s}, 1 \mathrm{H})$, 4.99 (s, 1H), 4.20 (t, $J=7.1 \mathrm{~Hz}, 1 \mathrm{H}), 3.92(\mathrm{~d}, J=7.1 \mathrm{~Hz}, 2 \mathrm{H}), 2.66-2.44(\mathrm{~m}, 4 \mathrm{H}), 1.72$ - $1.64(\mathrm{~m}, 4 \mathrm{H}), 1.39(\mathrm{~s}, 3 \mathrm{H}), 1.33(\mathrm{~s}, 3 \mathrm{H}), 1.29$ - $1.16(\mathrm{~m}, 2 \mathrm{H}) ;{ }^{13} \mathrm{C}$ NMR $(75 \mathrm{MHz}$, $\left.\mathrm{CDCl}_{3}\right): \delta 147.3,139.4,128.38,128.37,128.36,128.32,125.7,110.0,108.5,104.1$, 87.2, 85.3, 81.8, 68.0, 65.7, 36.5, 32.5, 28.36, 28.34, 24.7, 15.2; IR (neat) $\mathrm{cm}^{-1}: 3350$; MS (EI): $m / z 290\left(\mathrm{M}^{+}\right)$; HRMS (EI) calcd for $\mathrm{C}_{18} \mathrm{H}_{26} \mathrm{O}_{3}: 290.1882\left(\mathbf{M}^{+}\right)$, found: 290.1880 .

\section{References}

(1) Matsuya, Y.; Itoh, T.; Nemoto, H. Eur. J. Org. Chem. 2003, 2221-2224. 


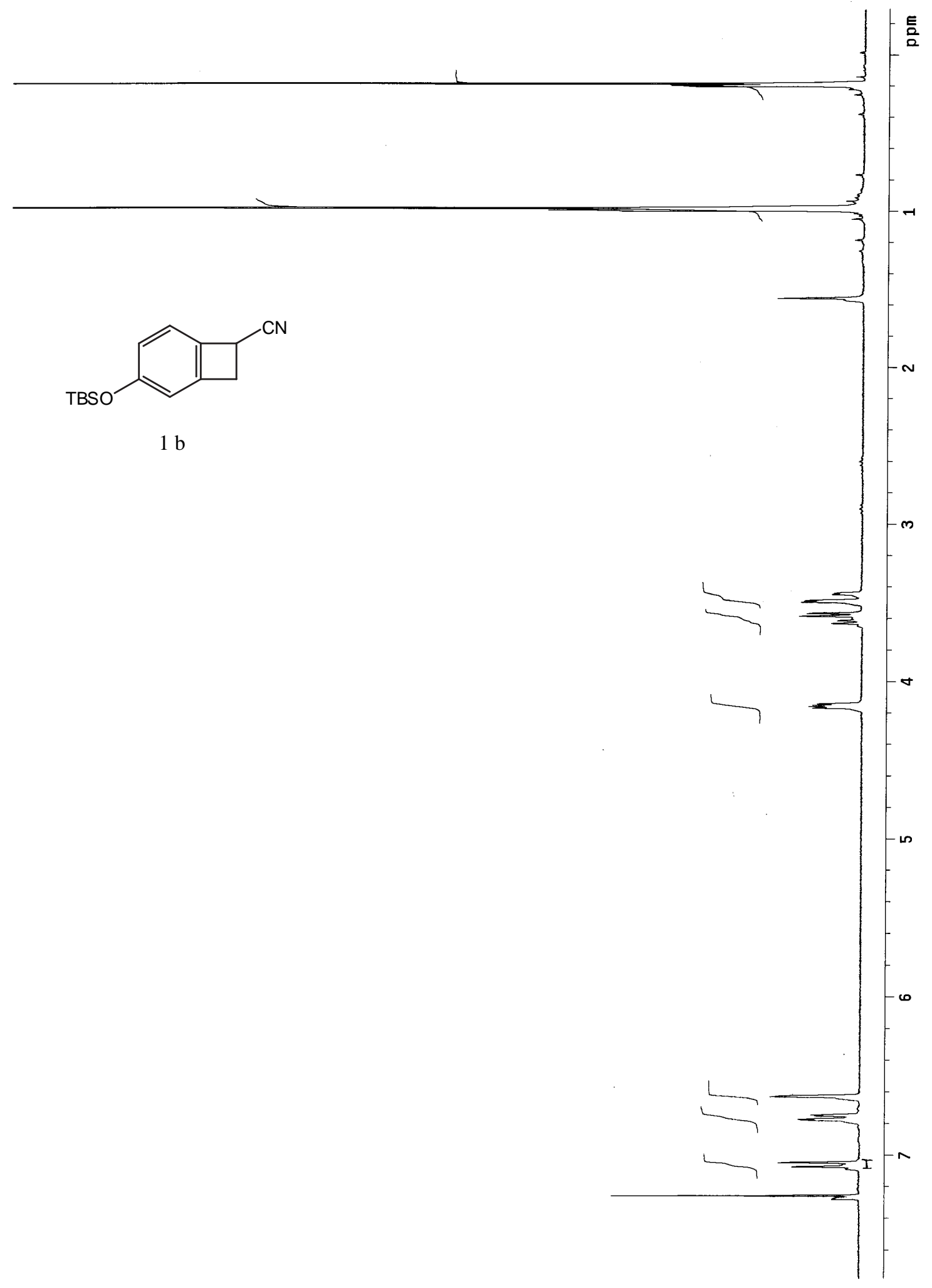




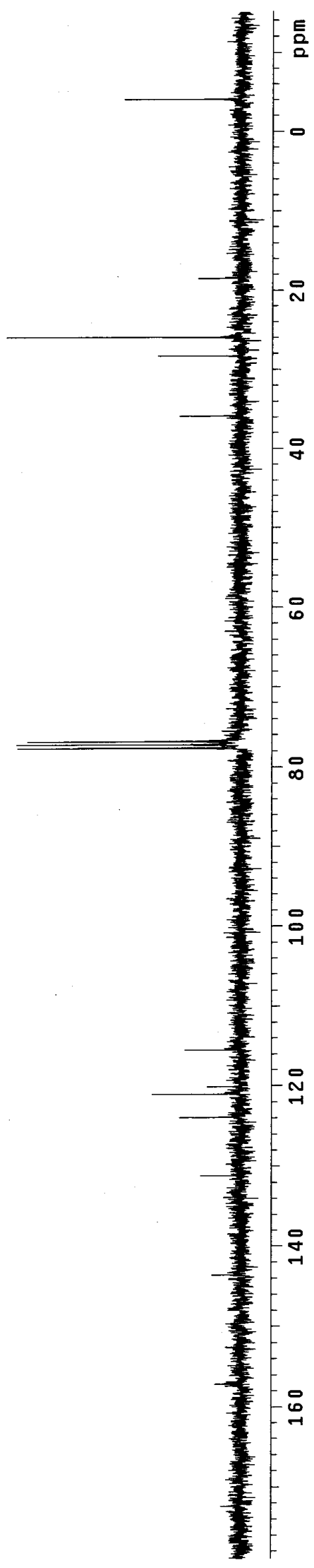



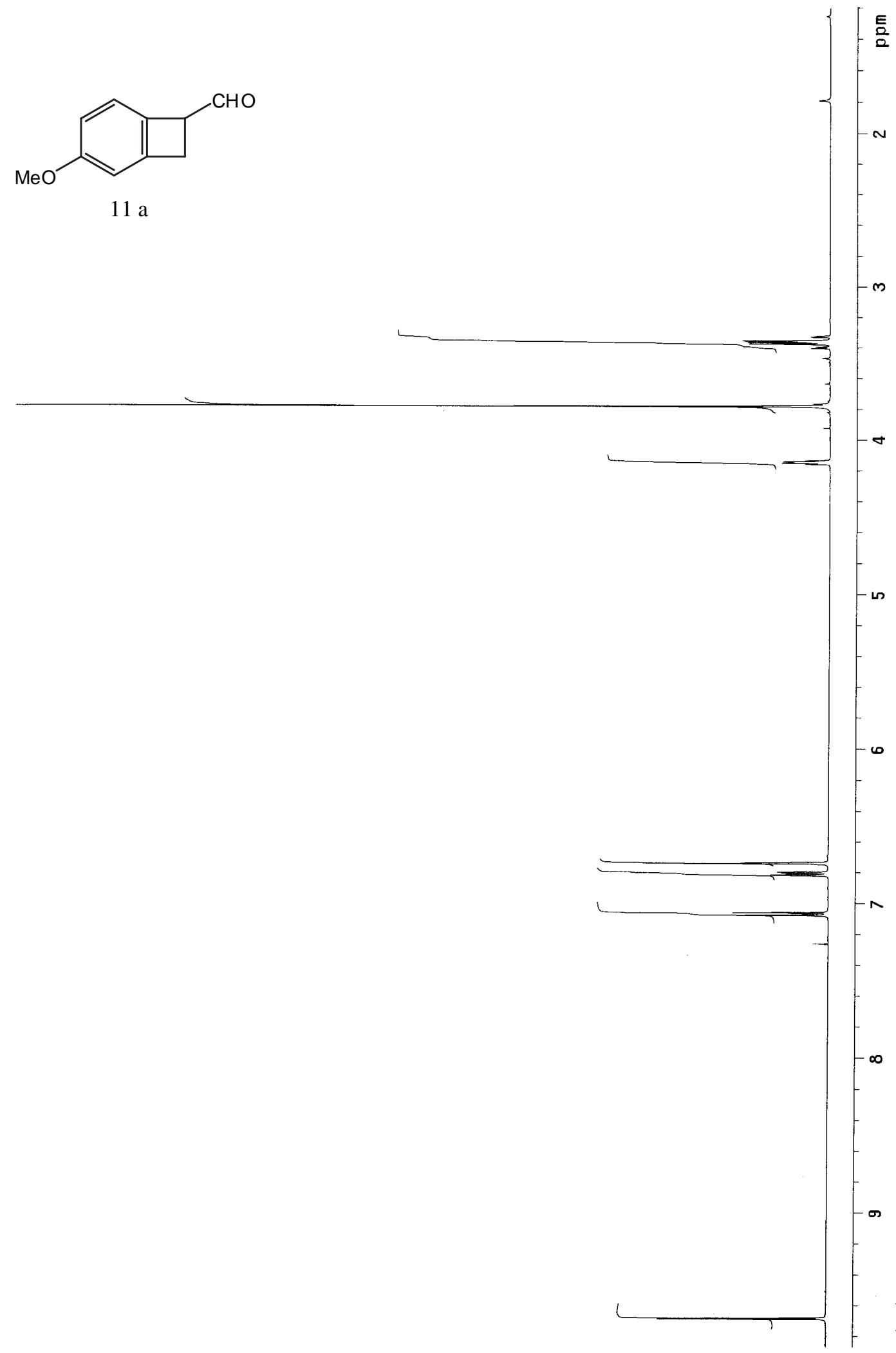


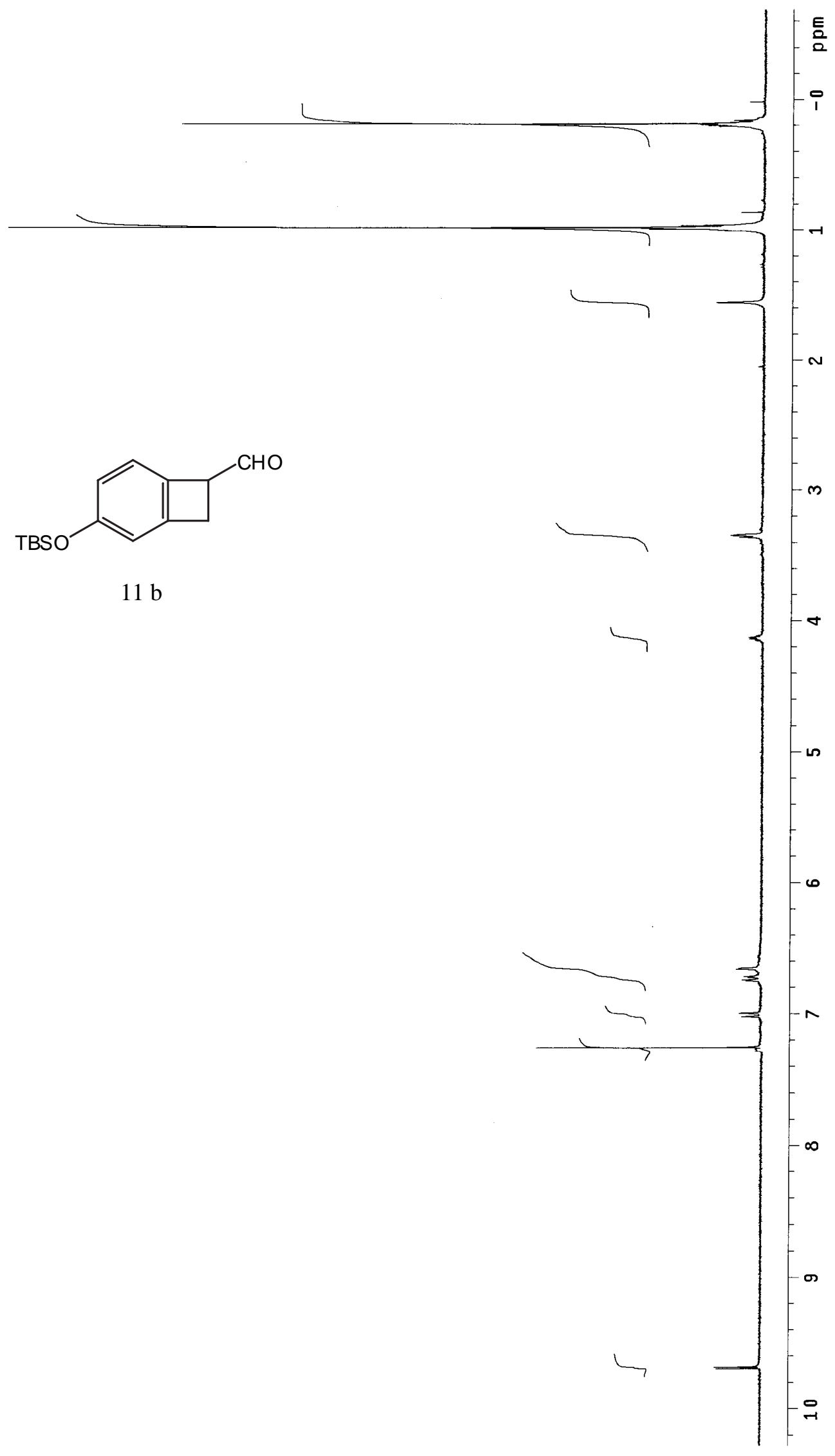



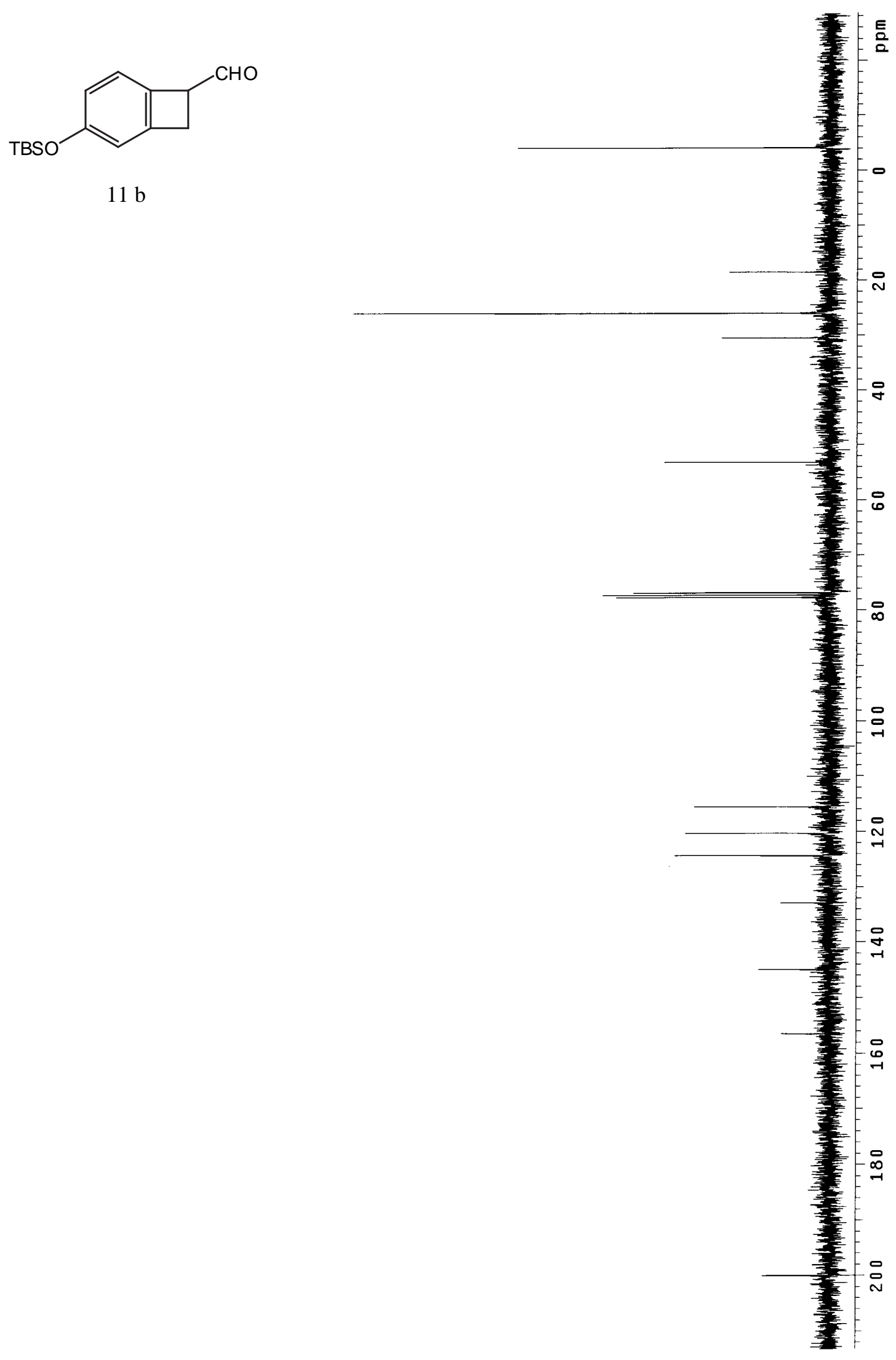

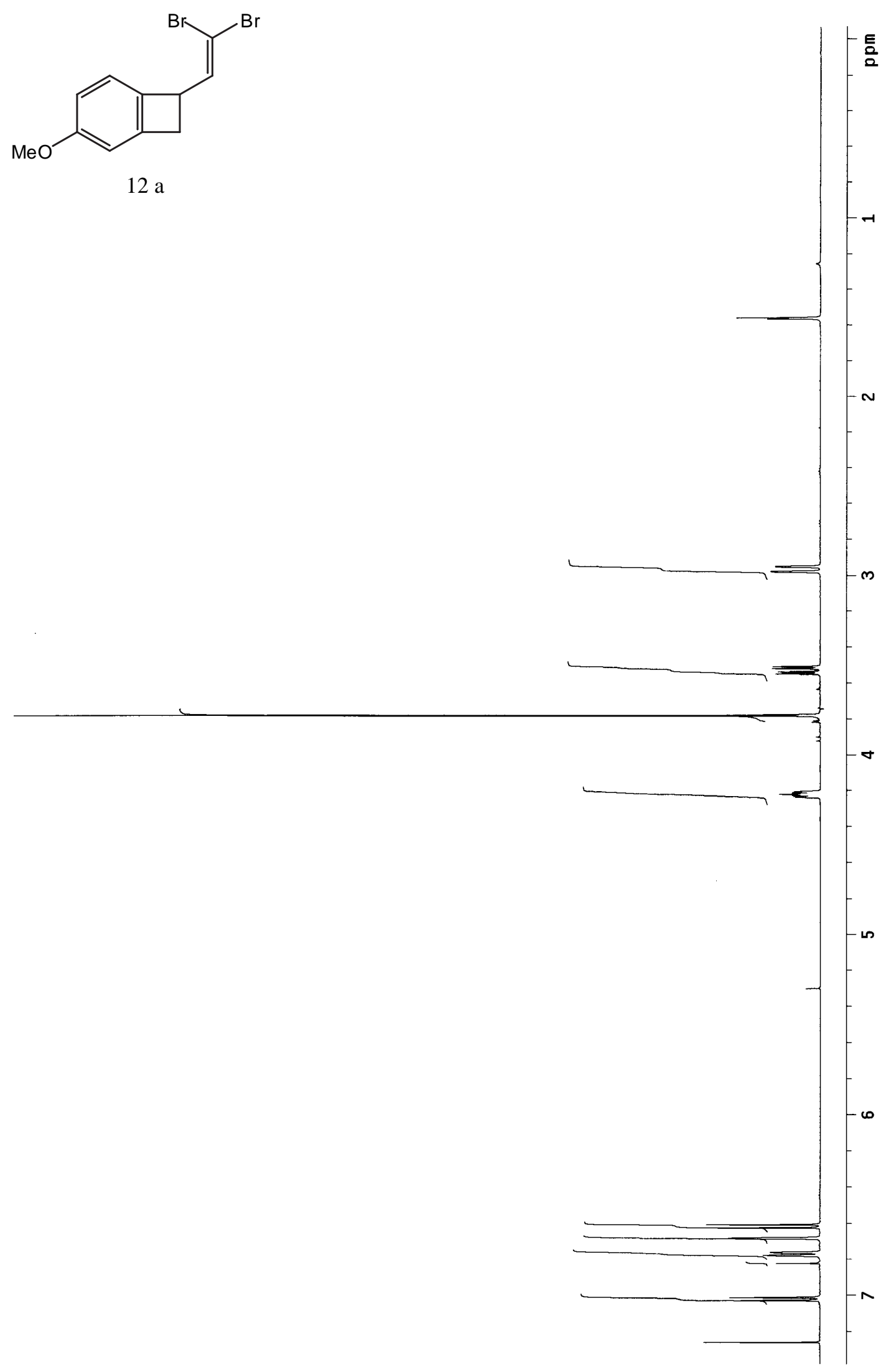

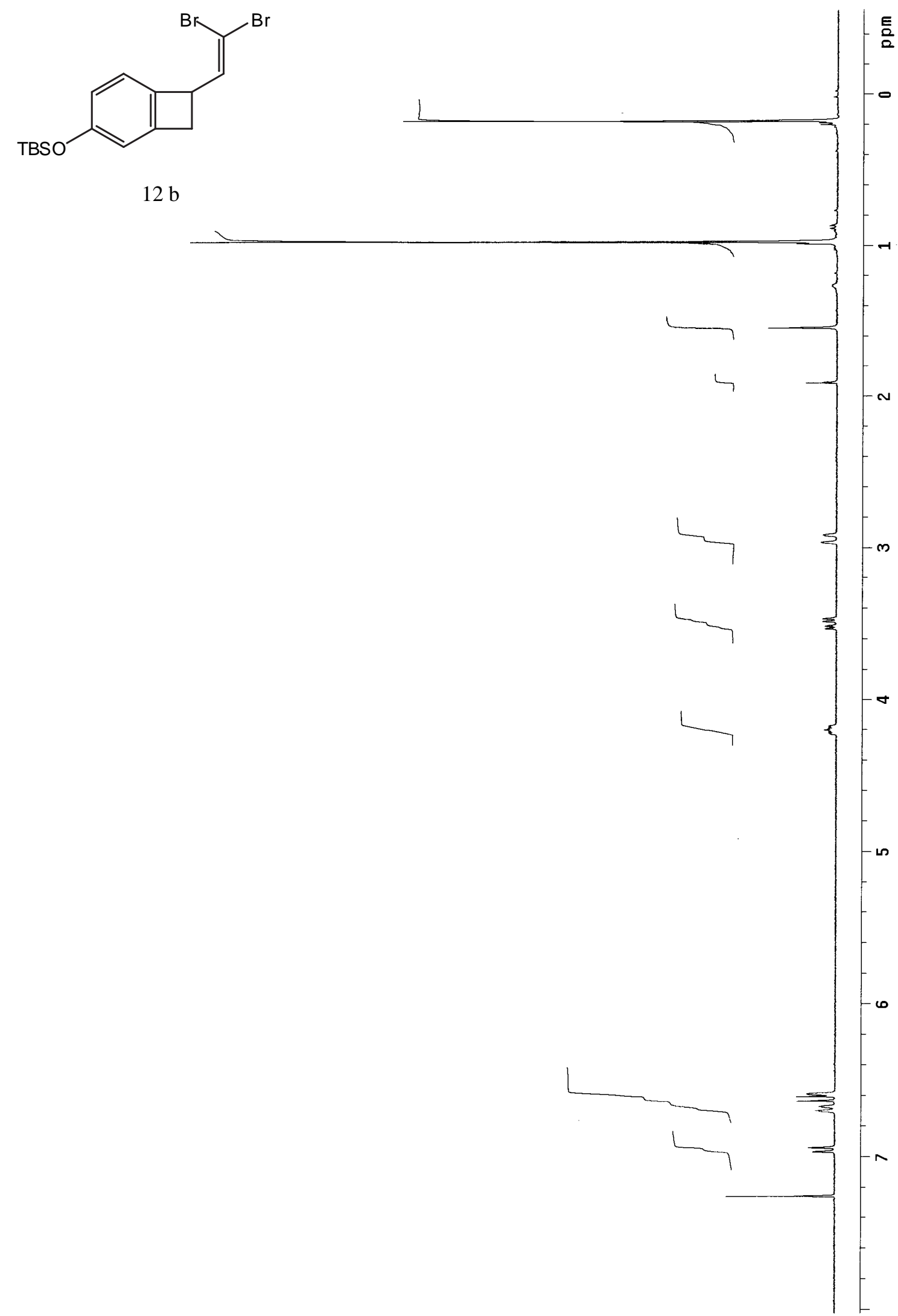

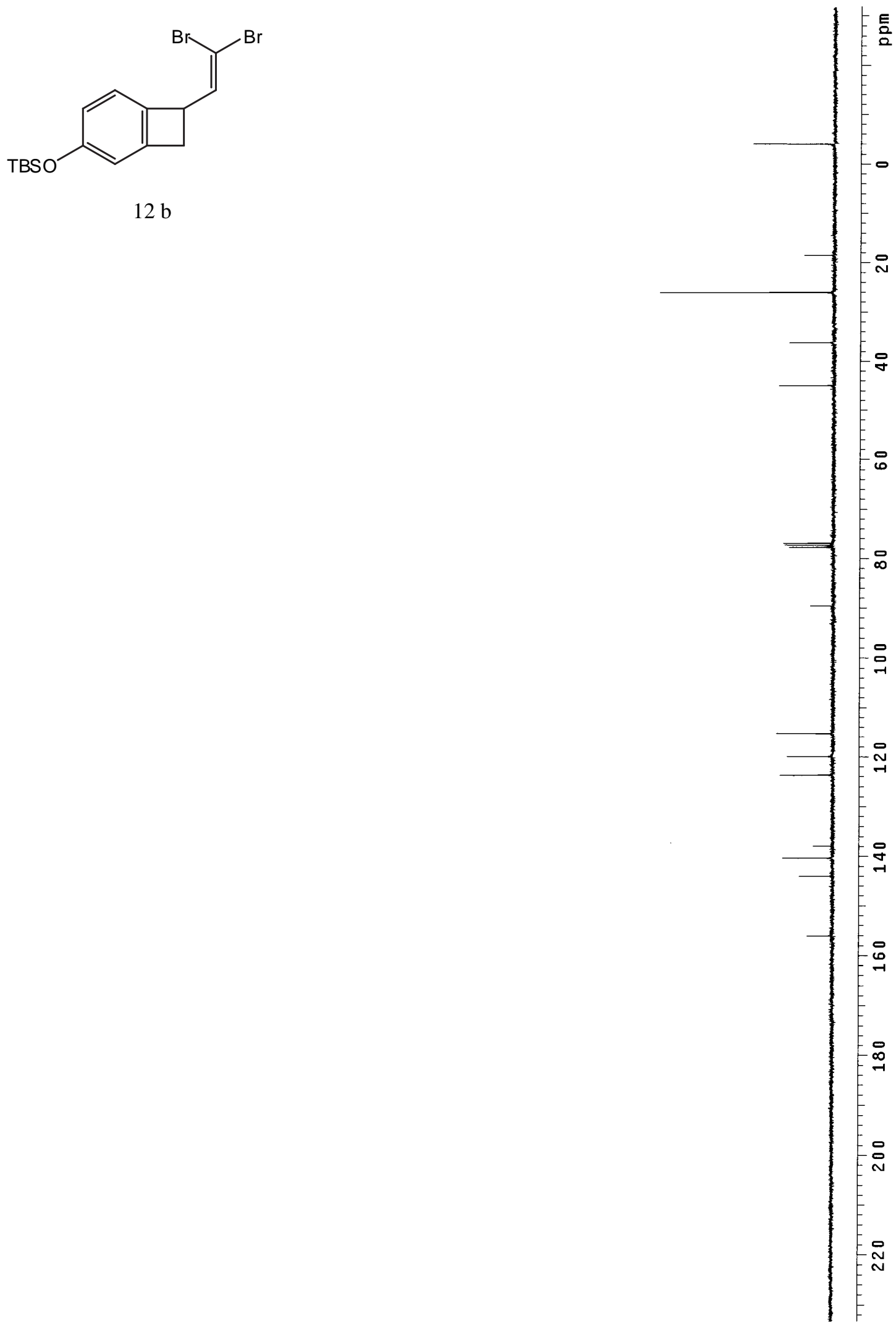


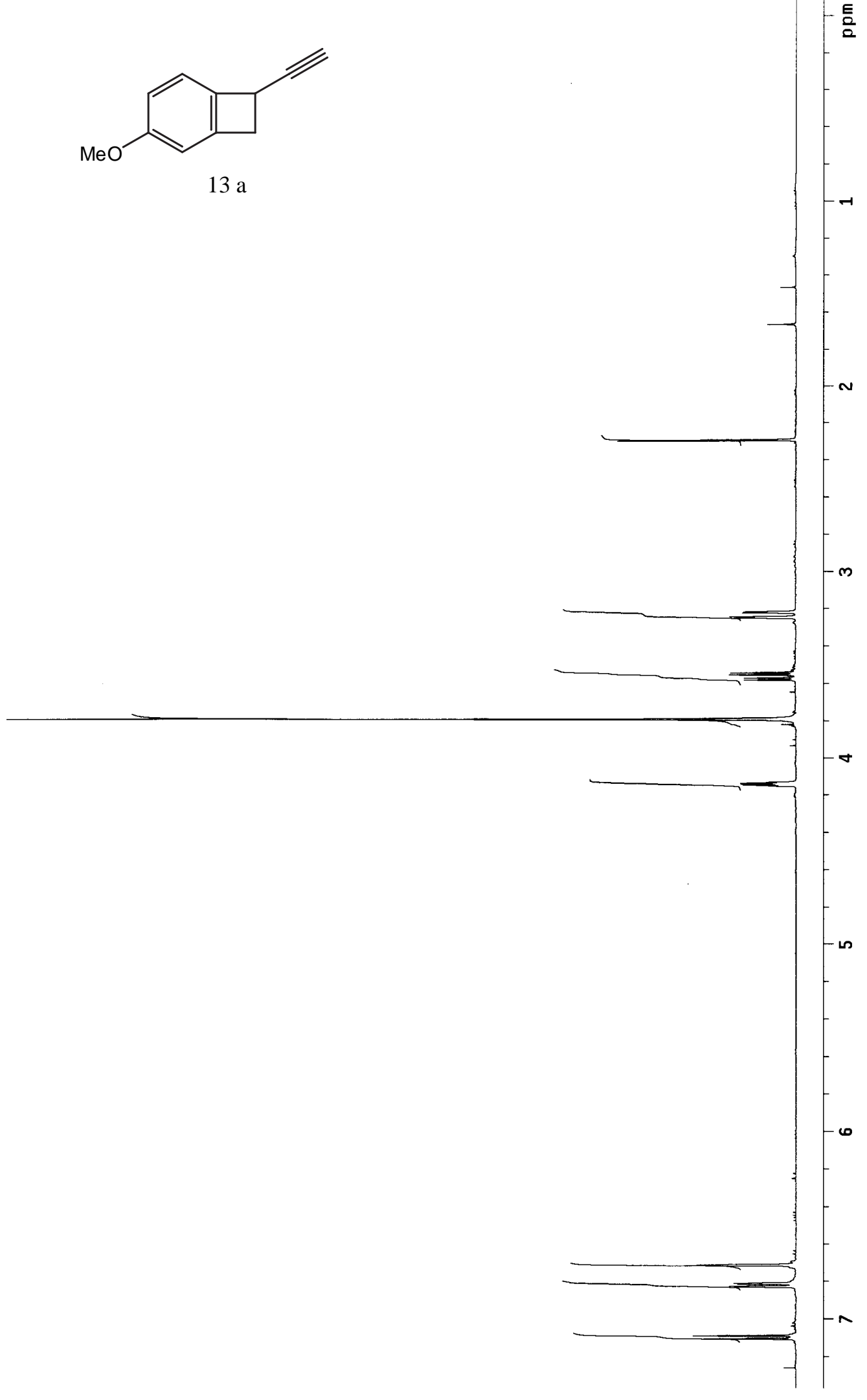




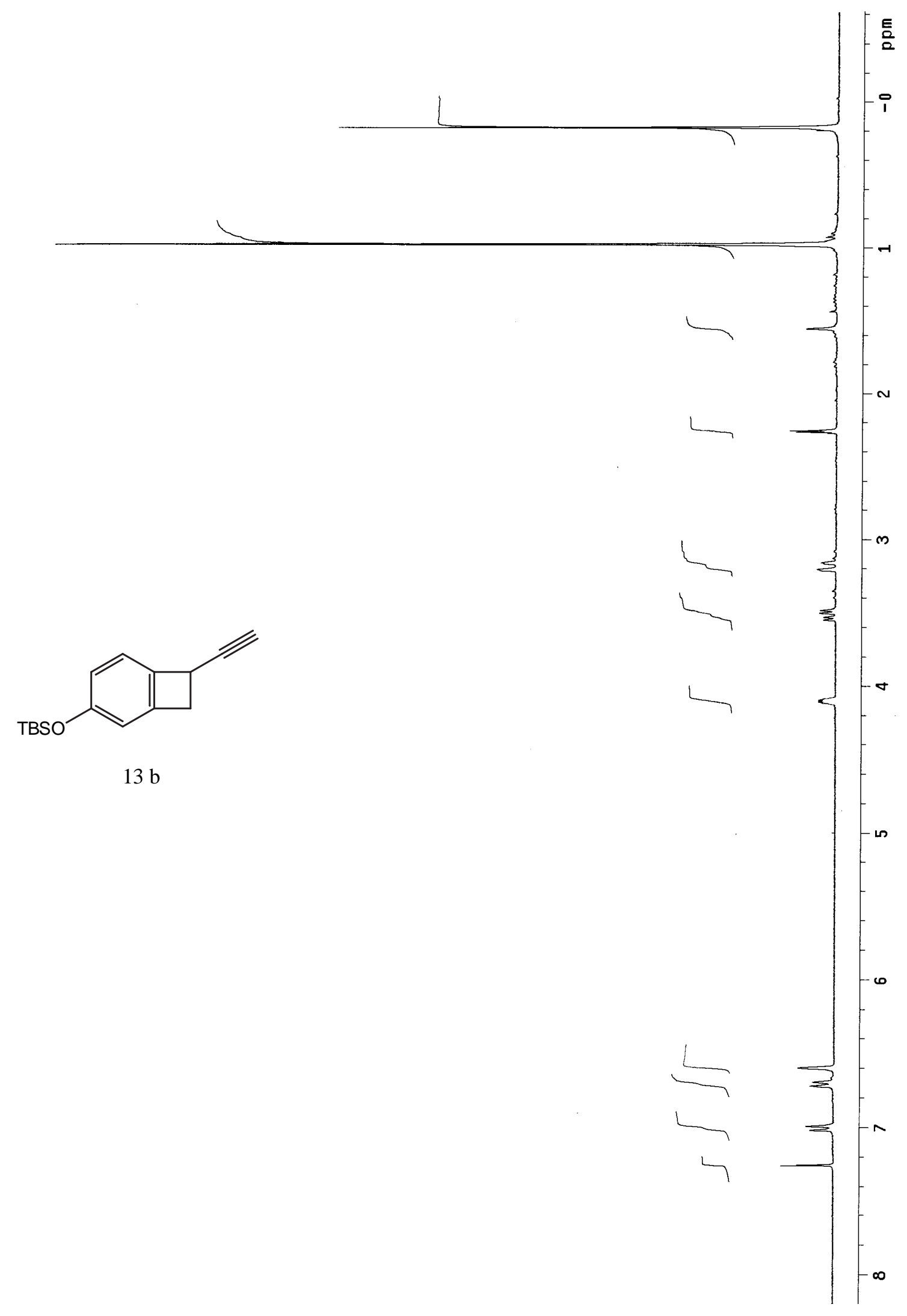




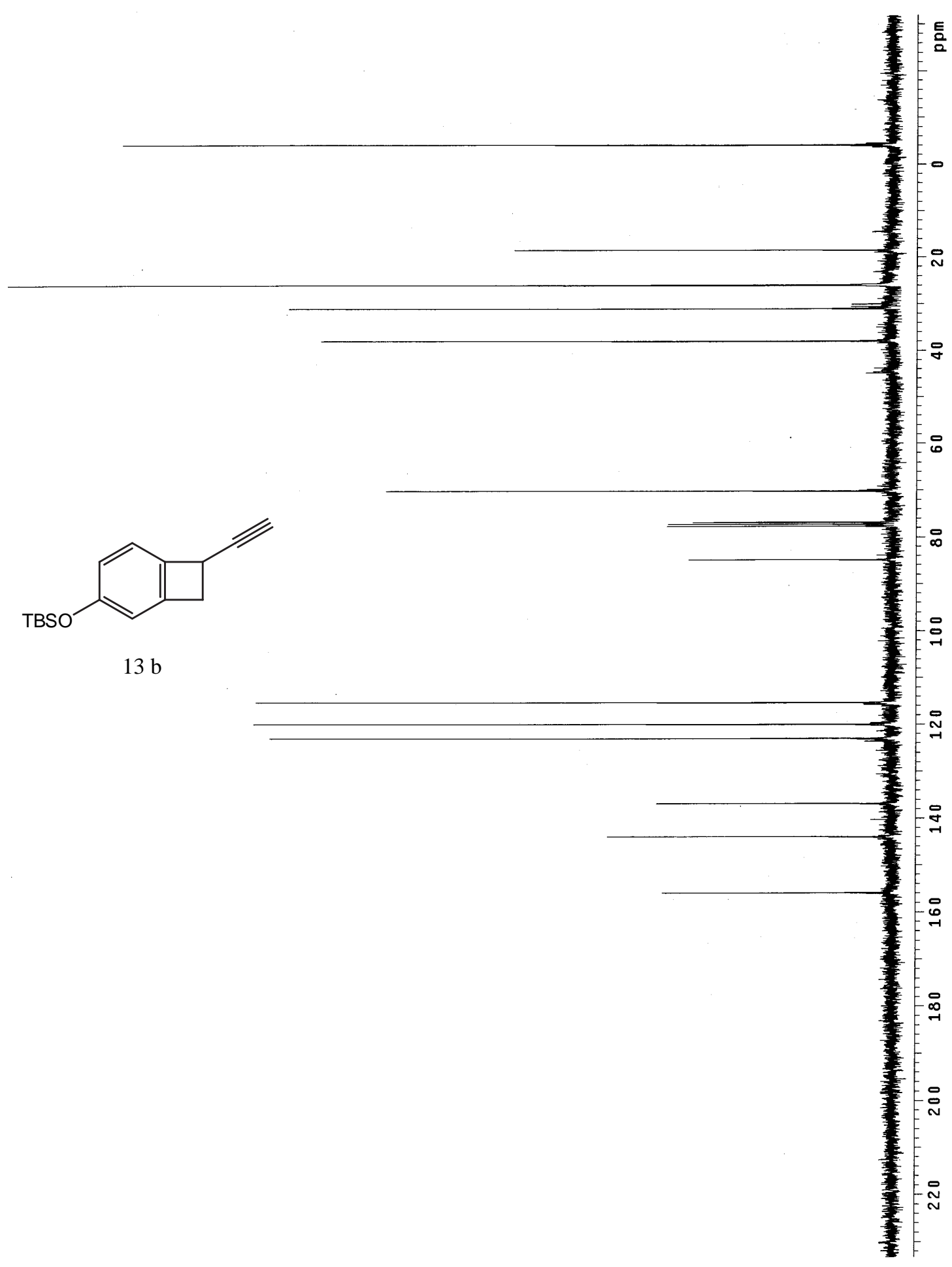




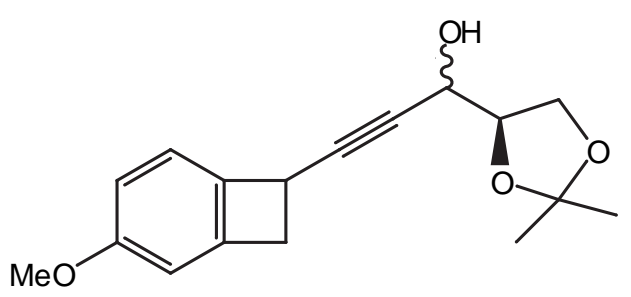

$14 \mathrm{a}$

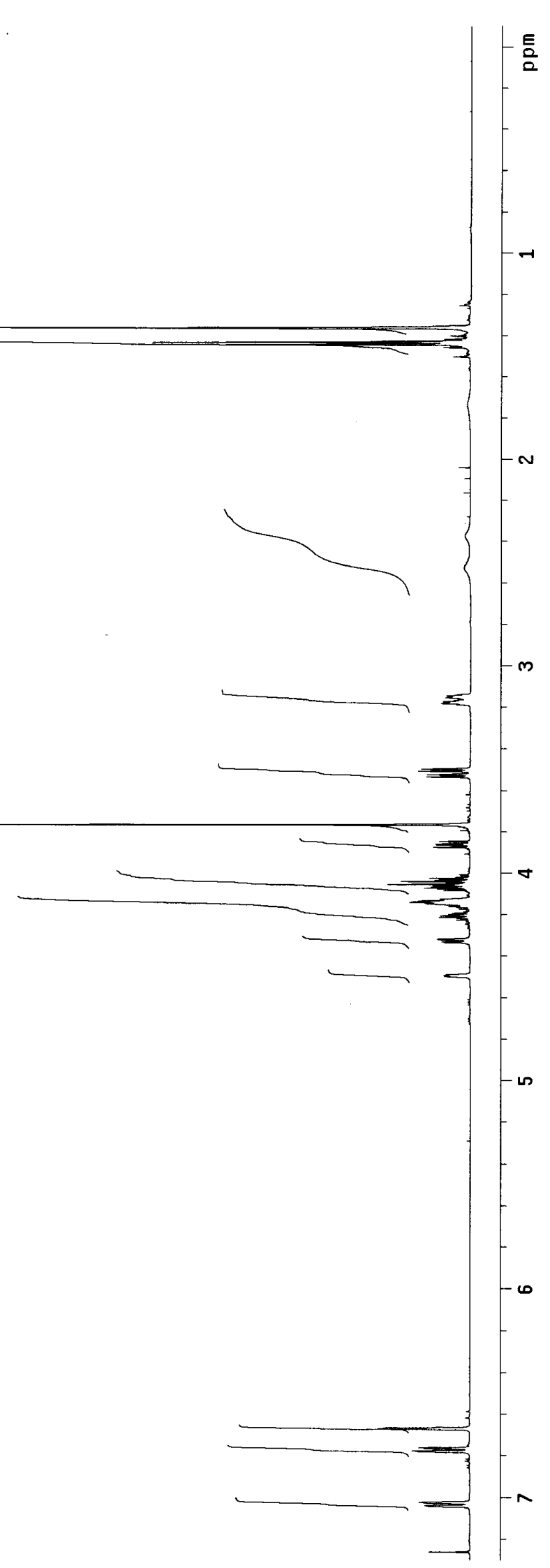




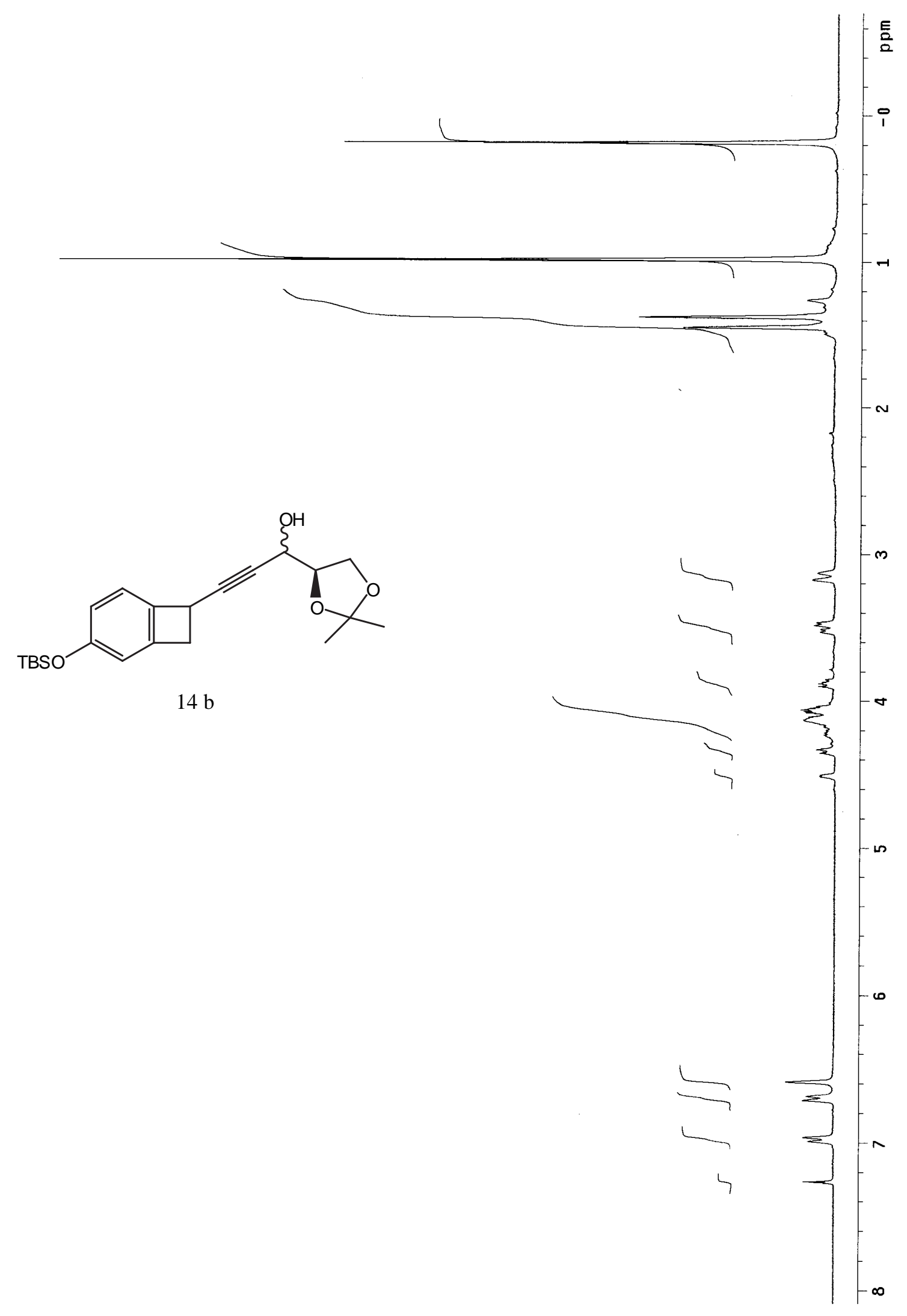




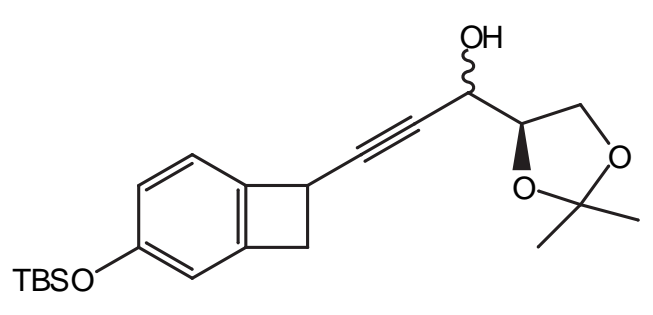

$14 \mathrm{~b}$

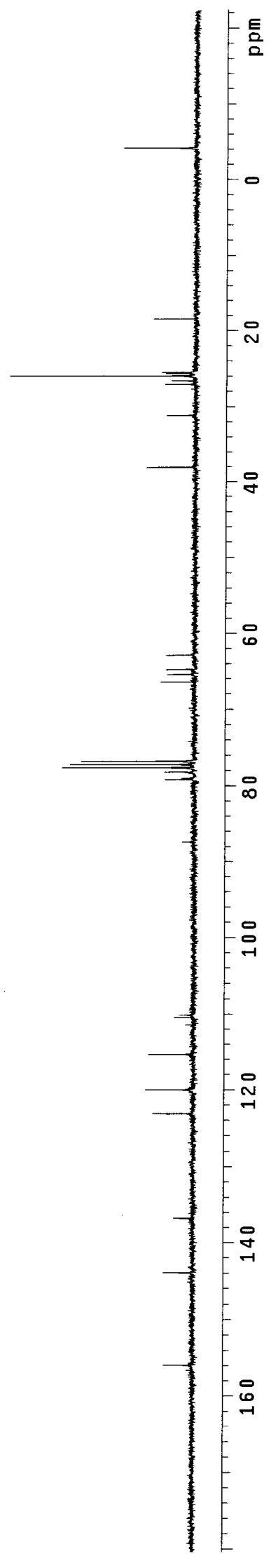

S21 


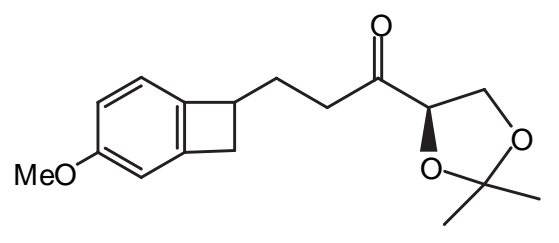

틈

2 a (from 14 a)

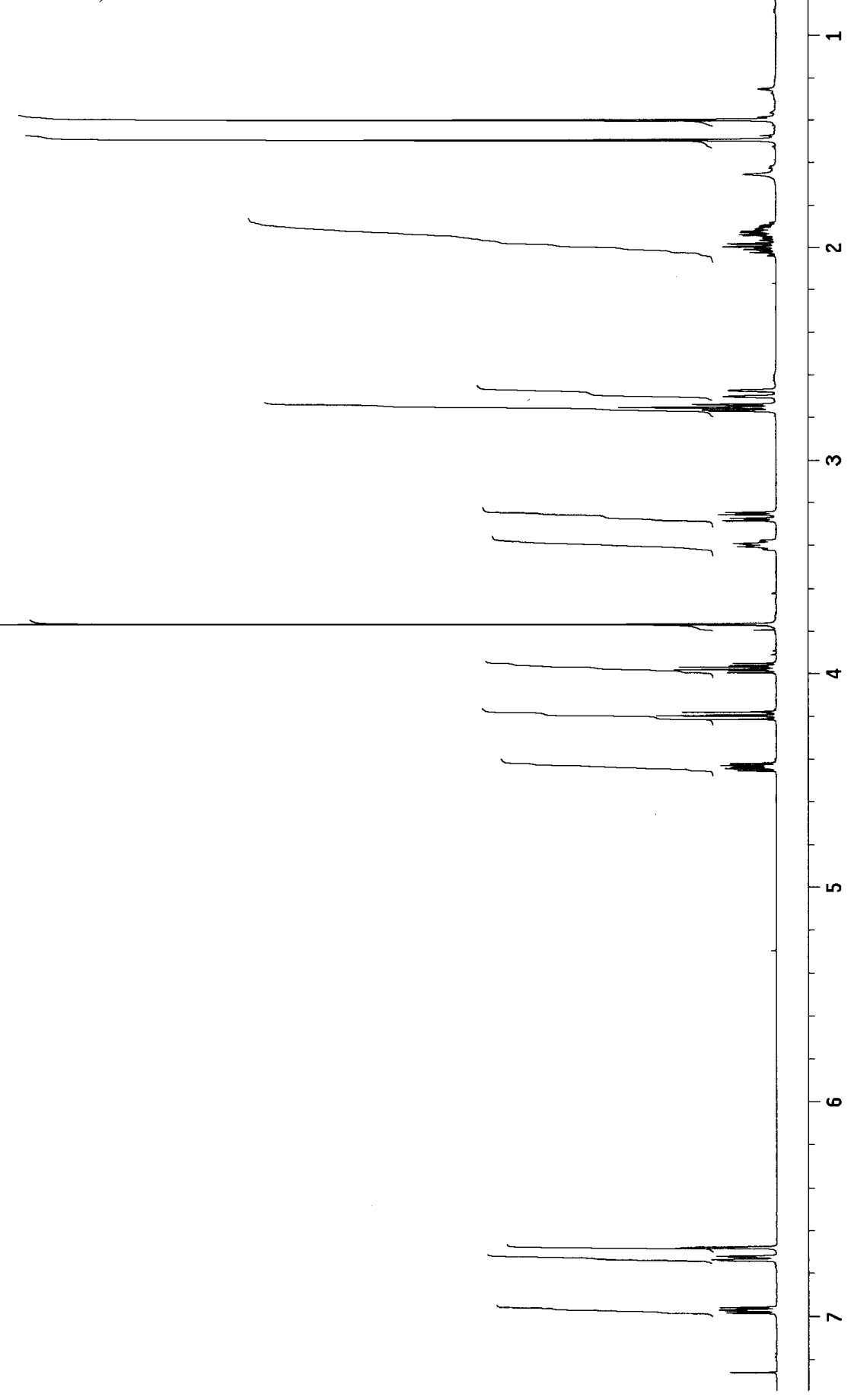




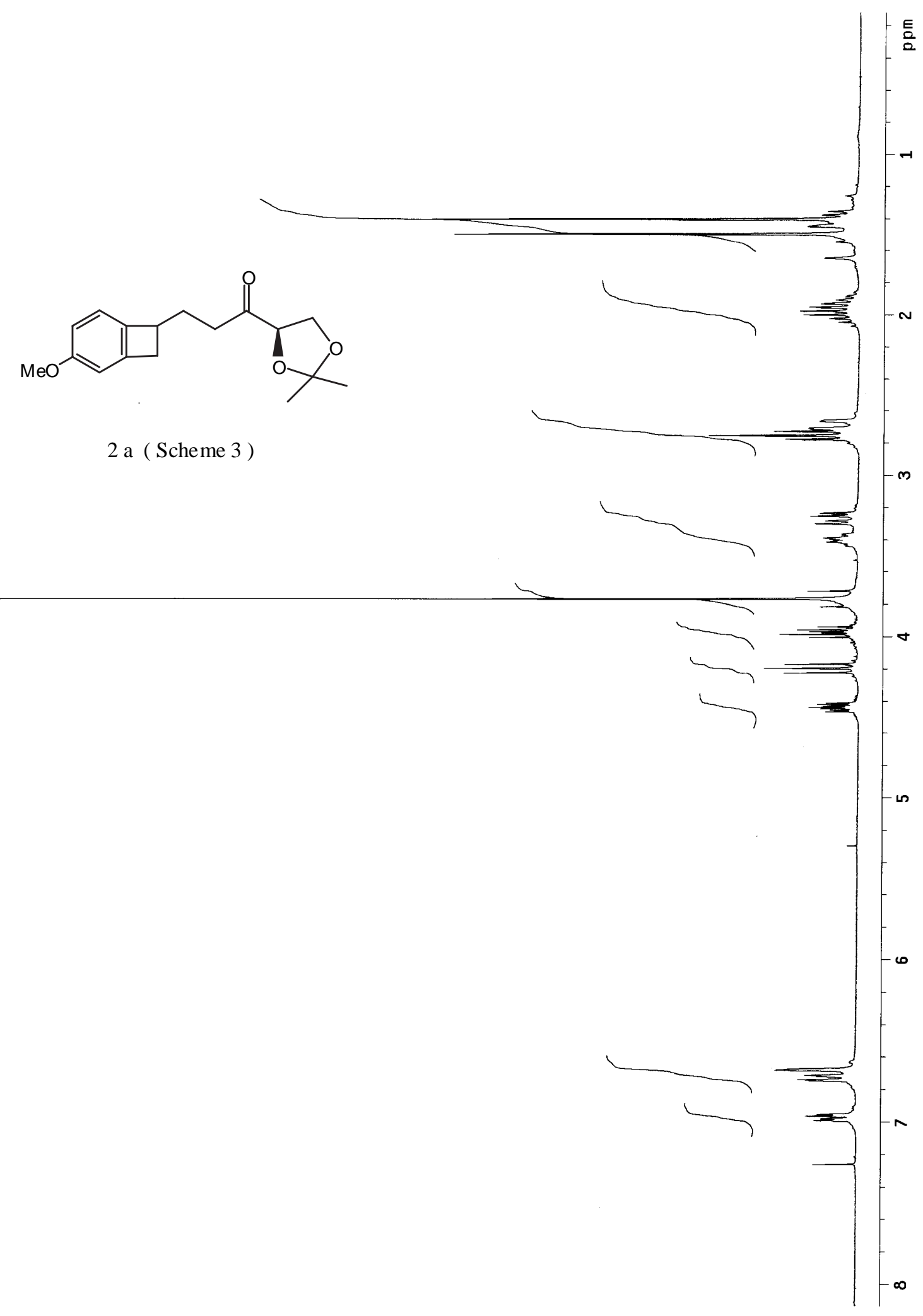




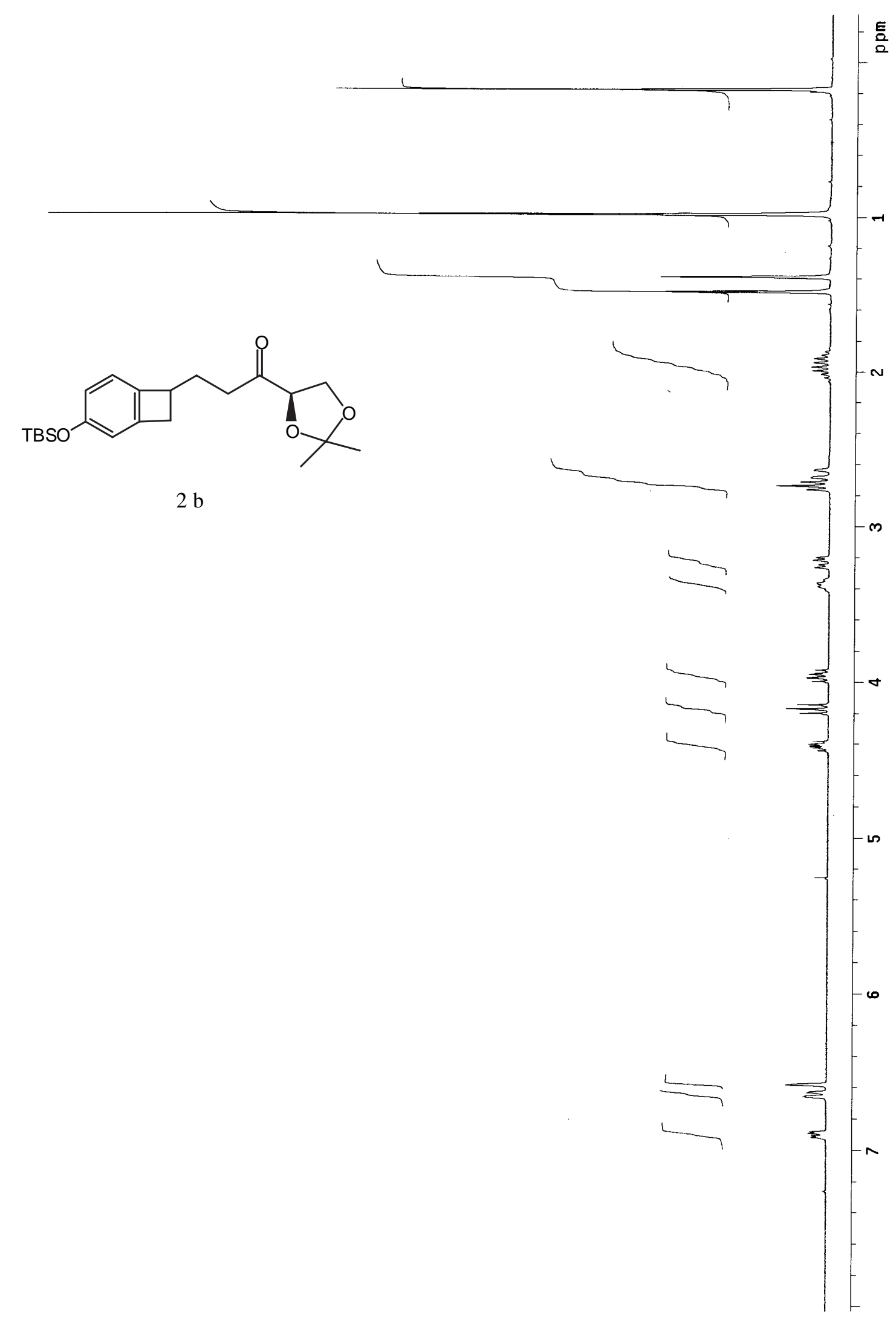




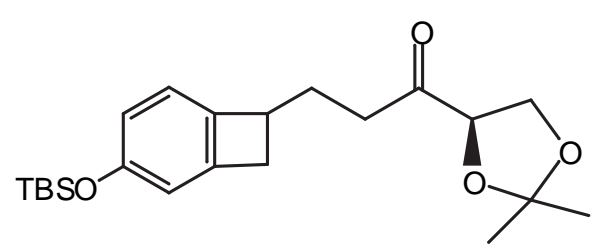

2 b

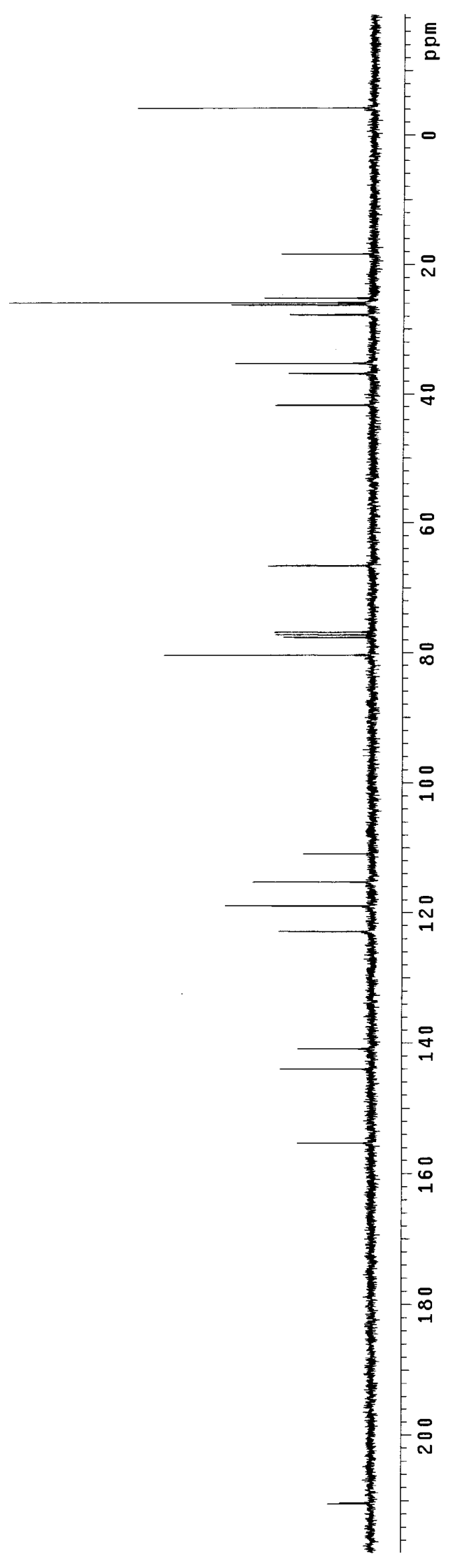

S25 


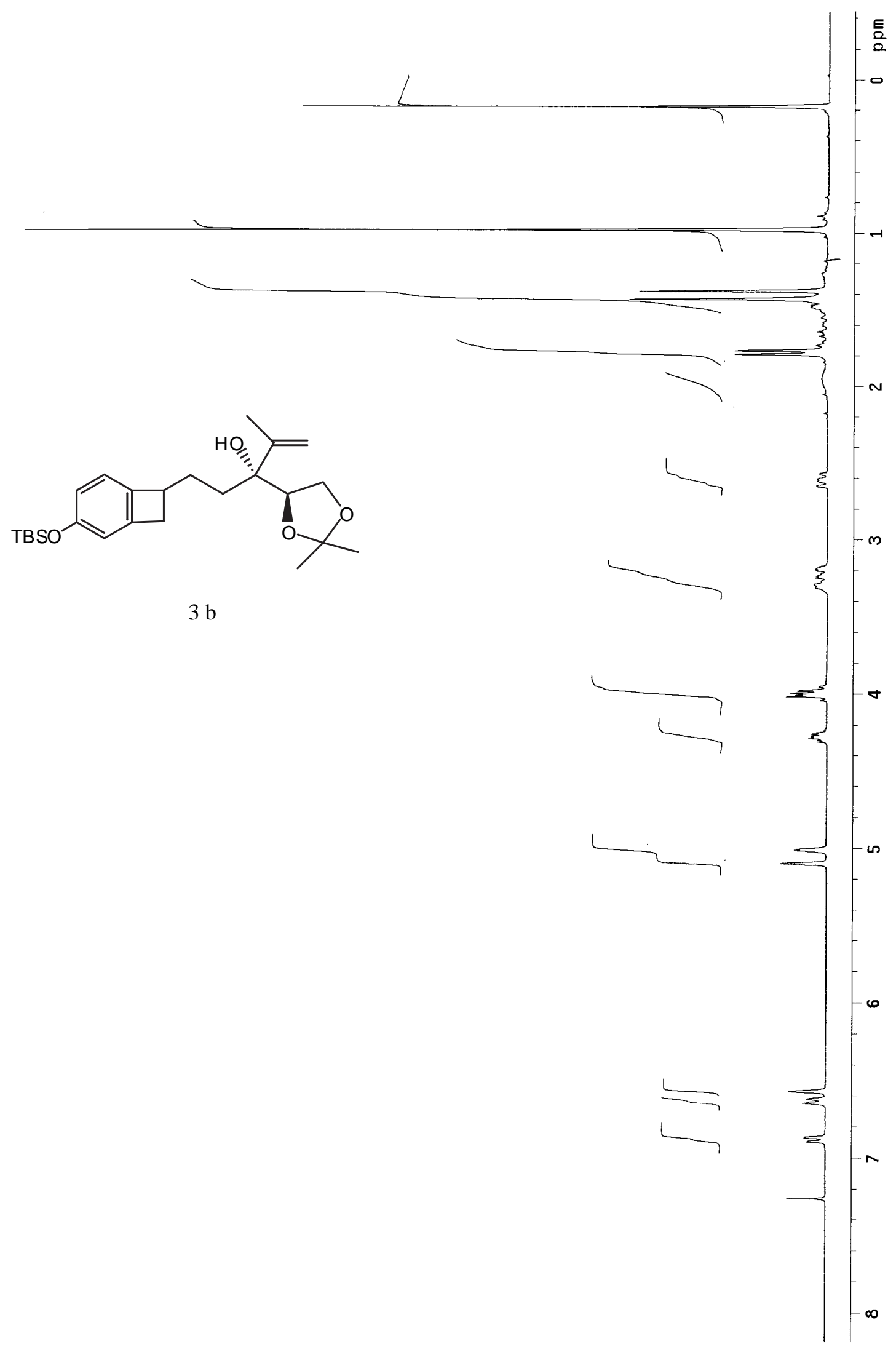



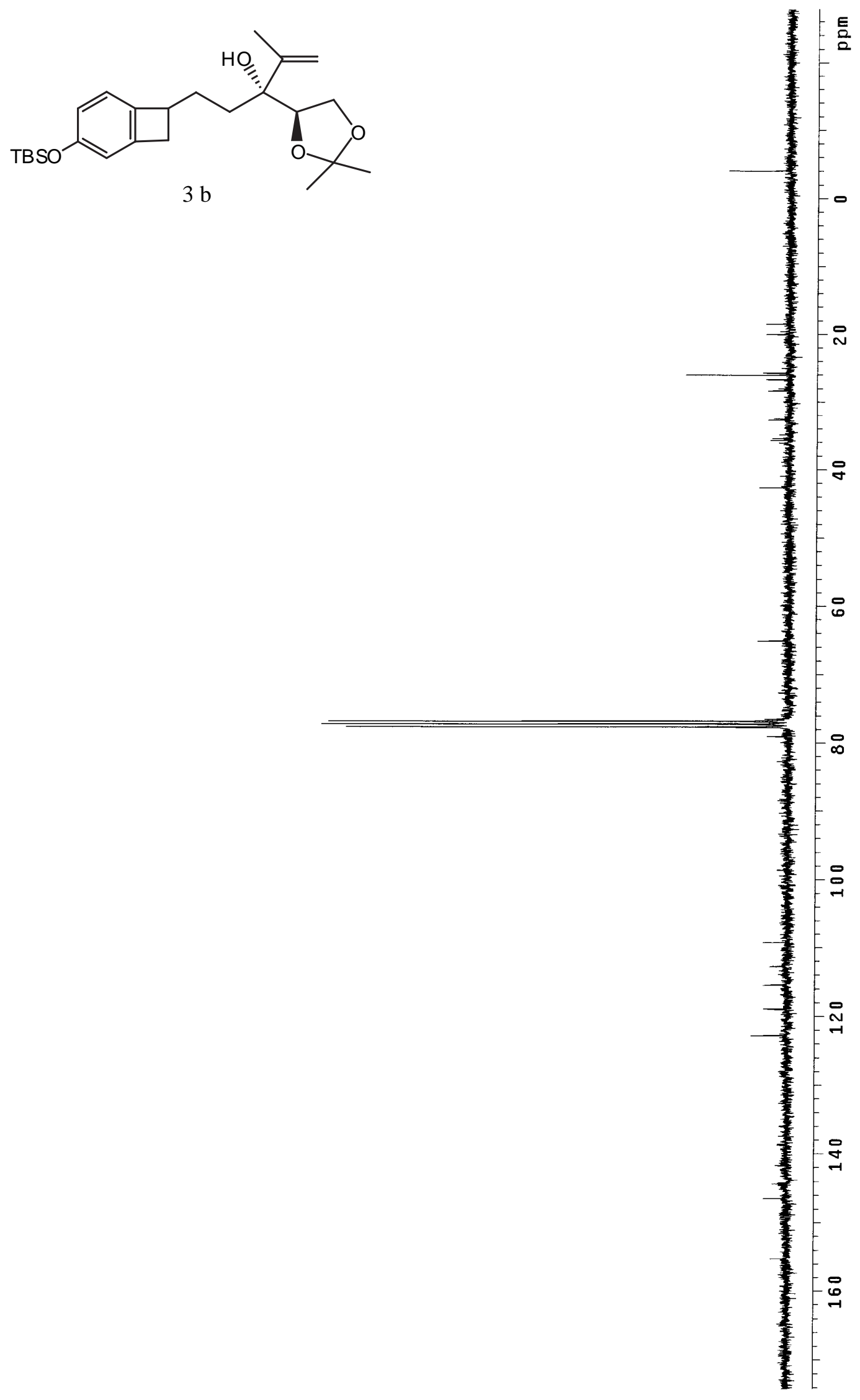


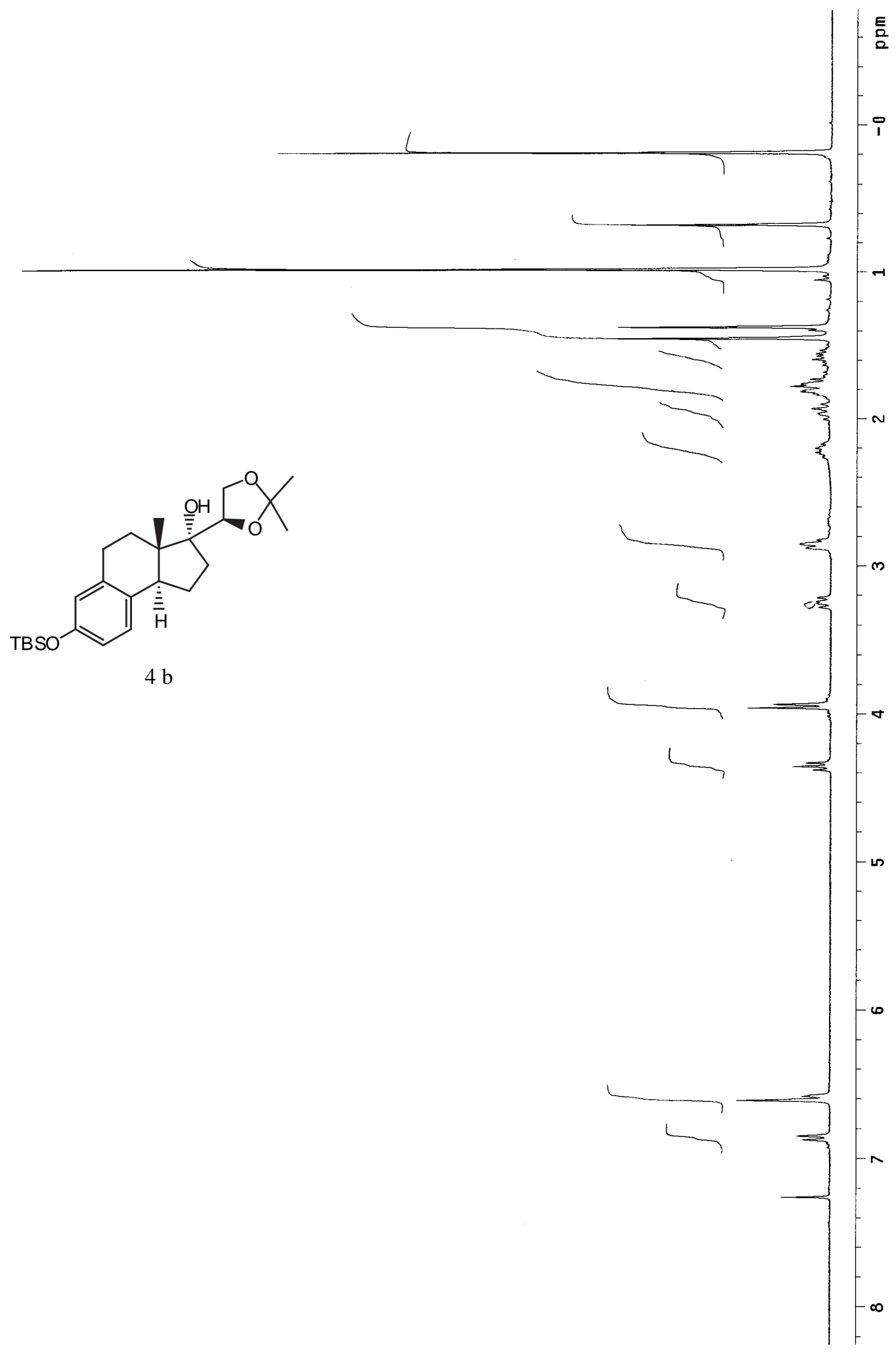




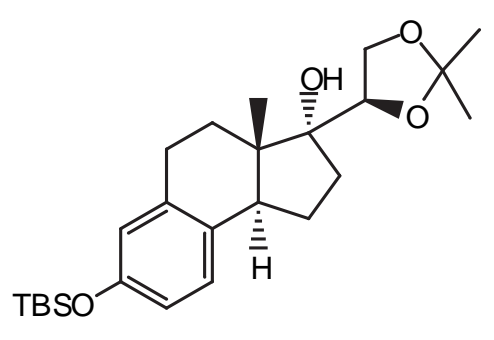

$4 \mathrm{~b}$

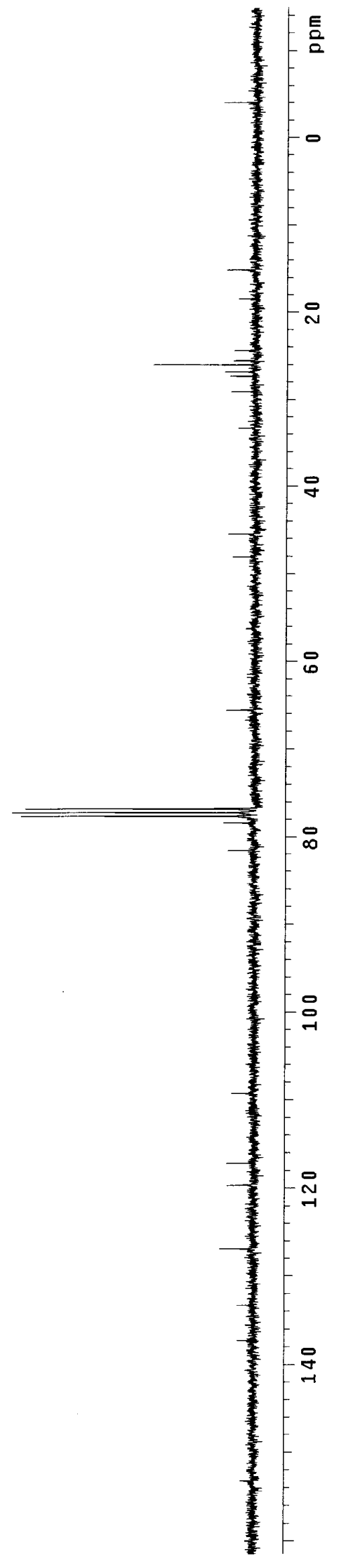

S29 


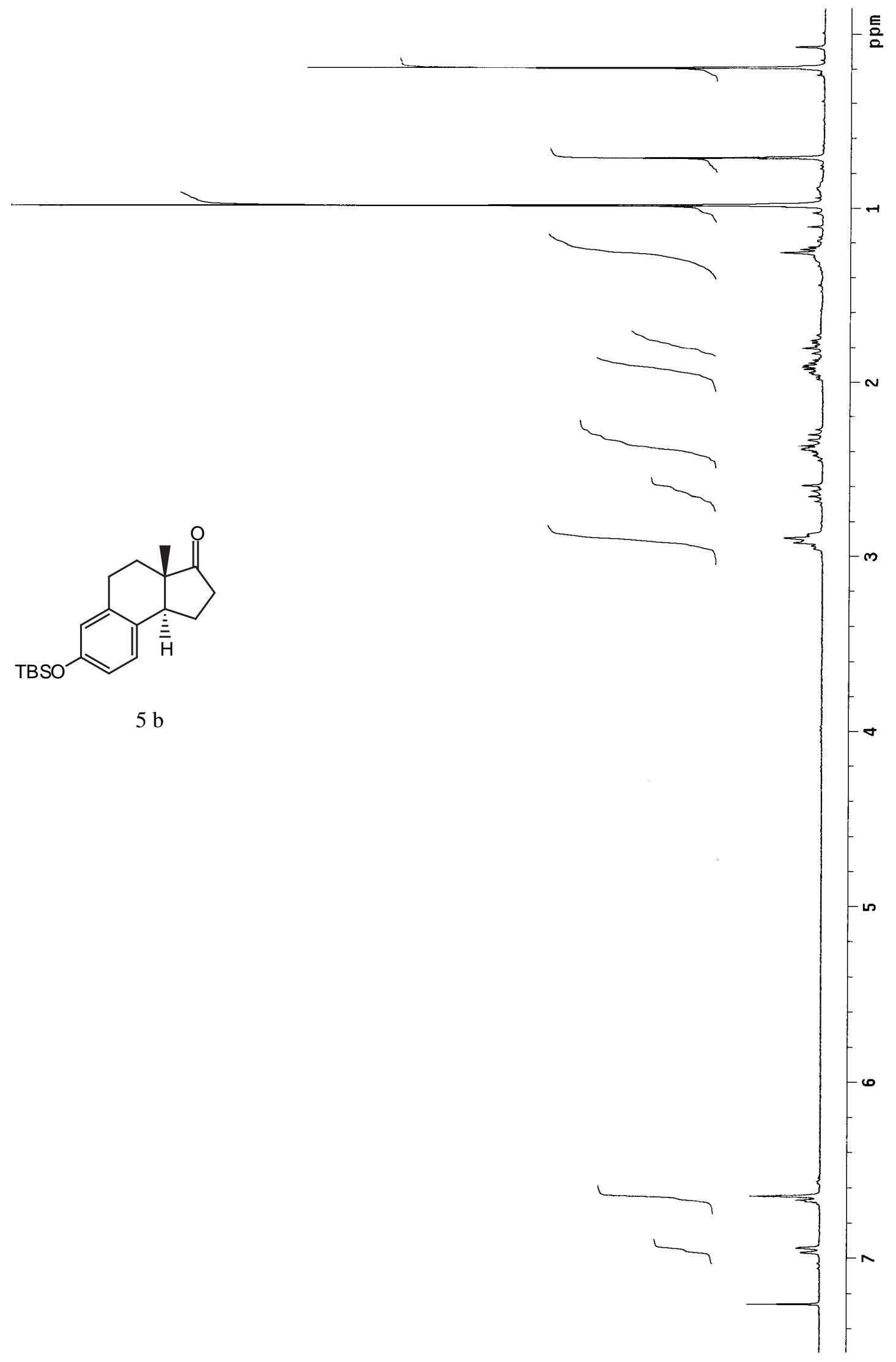




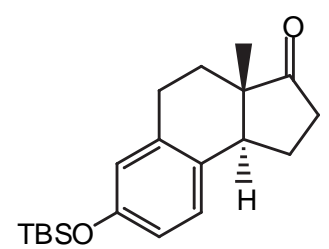

$5 \mathrm{~b}$

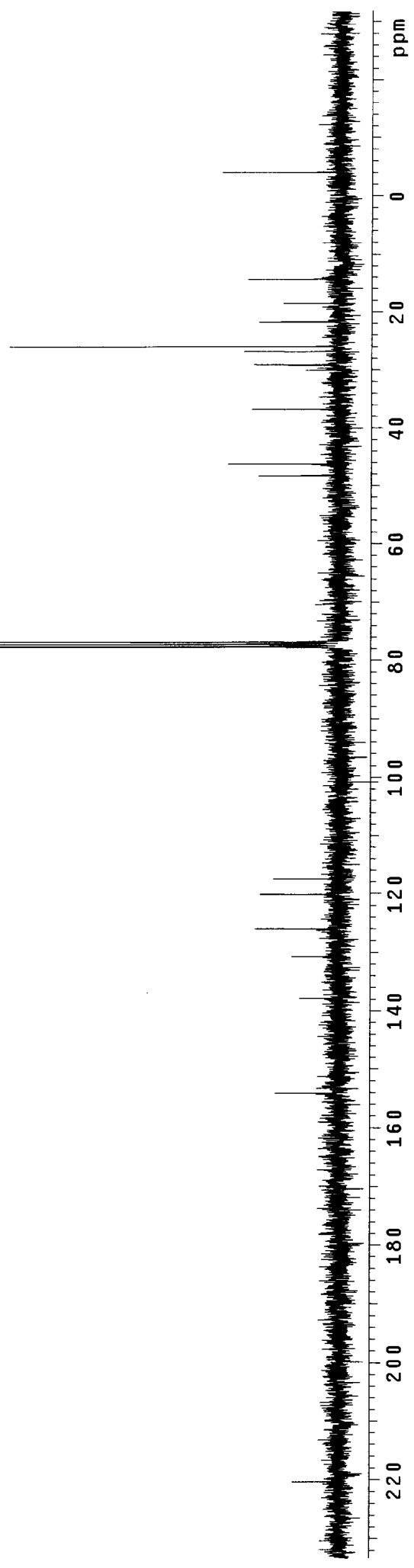




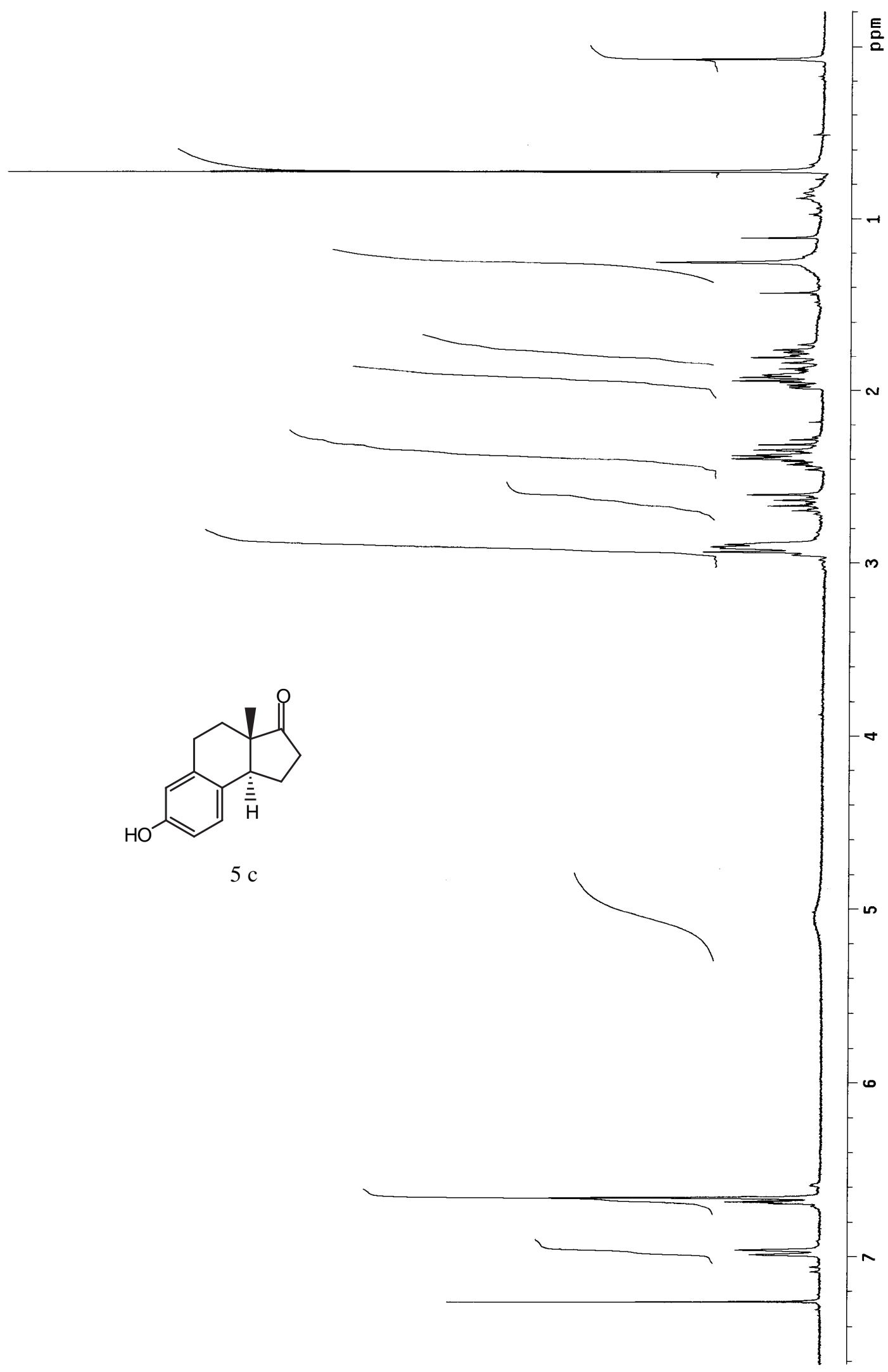



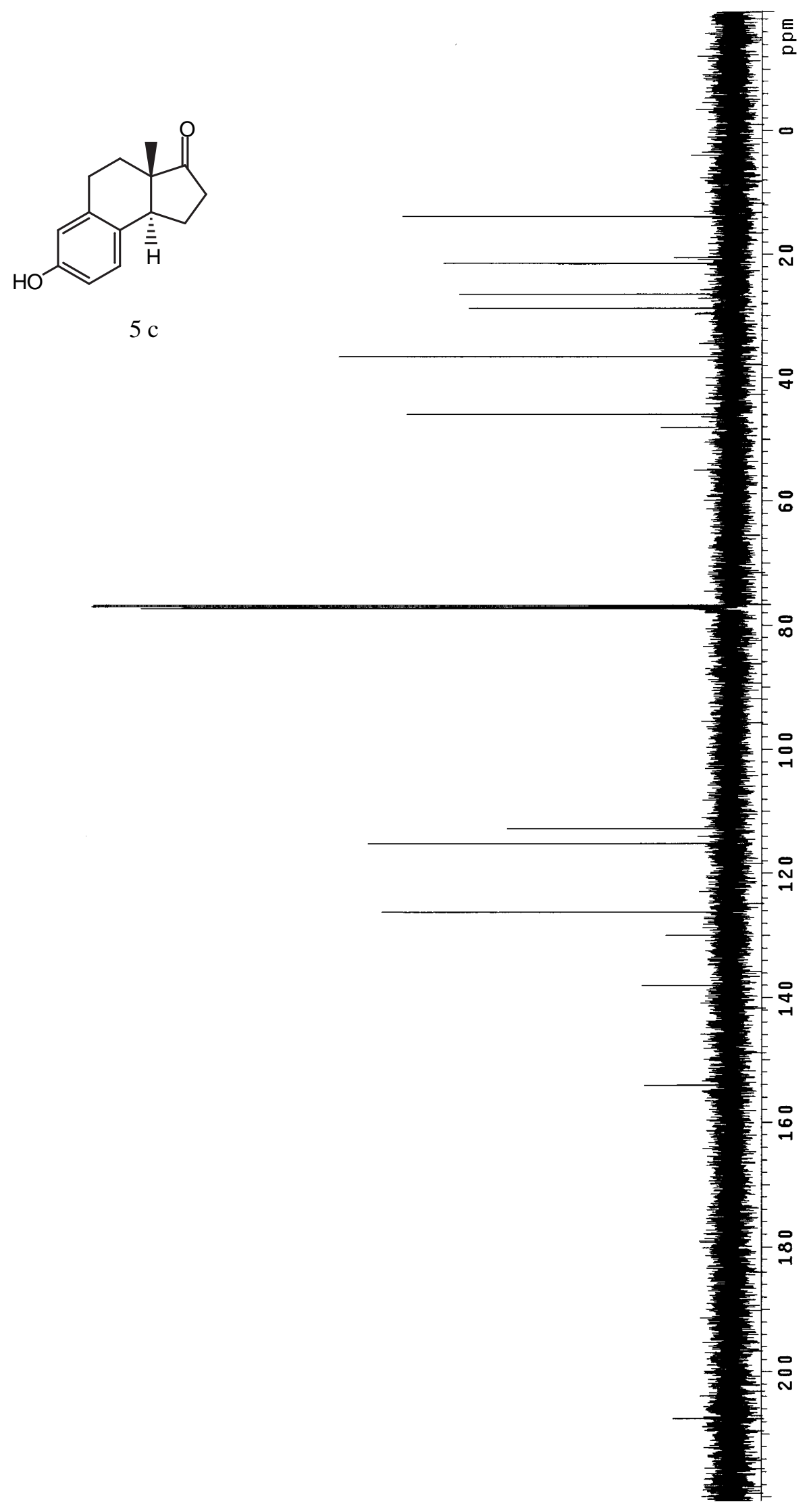

S33 